\title{
Electronic Relaxation Dynamics in 2-Quinolinones with Extended Conjugation
}

\author{
Alana Fligelman, Gonto Johns, Christina Guyn, Alexis Petrauskas, Paul A. \\ Vadola, and Graham B. Griffin* \\ Department of Chemistry and Biochemistry, DePaul University, $1110 \mathrm{~W}$ Belden Ave, Chicago \\ IL, 60614, USA. \\ *corresponding author: phone (773)325-7099, fax (773)325-7421, ggriffi6@ depaul.edu
}


Table of Contents

\begin{tabular}{|c|c|}
\hline ITEM & PAGE \\
\hline 1. Electronic Structure Calculation Results & 3 \\
\hline $\begin{array}{l}\text { Table S1: Energies from Electronic Structure Calculations at the Optimized Ground } \\
\text { State Geometry }\end{array}$ & 3 \\
\hline $\begin{array}{l}\text { Table S2: Excited State Calculation Results for Compound } 1 \text { at the Optimized } S_{0} \\
\text { Geometry }\end{array}$ & 4 \\
\hline $\begin{array}{l}\text { Table S3: Excited State Calculation Results for Compound } 2 \text { at the Optimized } S_{0} \\
\text { Geometry }\end{array}$ & 5 \\
\hline $\begin{array}{l}\text { Table S4: Excited State Calculation Results for Compound } 3 \text { at the Optimized } S_{0} \\
\text { Geometry }\end{array}$ & 5 \\
\hline $\begin{array}{l}\text { Table S5: Excited State Calculation Results for Compound } 4 \text { at the Optimized } S_{0} \\
\text { Geometry }\end{array}$ & 6 \\
\hline $\begin{array}{l}\text { Table S6: Excited State Calculation Results for Compound } 5 \text { at the Optimized } S_{0} \\
\text { Geometry }\end{array}$ & 6 \\
\hline $\begin{array}{l}\text { Table S7: Excited State Calculation Results for Compound } 1 \text { at the Optimized } S_{1} \\
\text { Geometry }\end{array}$ & 7 \\
\hline $\begin{array}{l}\text { Table S8: Excited State Calculation Results for Compound } 2 \text { at the Optimized } S_{1} \\
\text { Geometry }\end{array}$ & 8 \\
\hline $\begin{array}{l}\text { Table S9: Excited State Calculation Results for Compound } 3 \text { at the Optimized } S_{1} \\
\text { Geometry }\end{array}$ & 8 \\
\hline $\begin{array}{l}\text { Table S10: Excited State Calculation Results for Compound } 4 \text { at the Optimized } S_{1} \\
\text { Geometry }\end{array}$ & 9 \\
\hline $\begin{array}{l}\text { Table S11: Excited State Calculation Results for Compound } 5 \text { at the Optimized } S_{1} \\
\text { Geometry }\end{array}$ & 9 \\
\hline 2. Vibronic Peak Progressions Observed in Some Absorbance Spectra & 10 \\
\hline Figure S1: Motion along normal coordinates for compounds 4 and 5 & 11 \\
\hline $\begin{array}{l}\text { Table S12: Predicted Vibrational Modes With Frequencies Near the Observed Vibronic } \\
\text { Energy Spacing }\end{array}$ & 12 \\
\hline 3. Transient Absorbance Spectra in Methanol Solution & 13 \\
\hline Figure S2: Waterfall plots of transient absorption data for all compounds & 13 \\
\hline 4. Fitting Kinetic Traces of Transient Absorption Data & 14 \\
\hline Figure S3: Transient Kinetic Fitting for Compound 1 in Methanol Solution & 15 \\
\hline Figure S4: Transient Kinetic Fitting for Compound 2 in Methanol Solution & 15 \\
\hline Figure S5: Transient Kinetic Fitting for Compound 3 in Methanol Solution & 16 \\
\hline Figure S6: Transient Kinetic Fitting for Compound 4 in Methanol Solution & 16 \\
\hline Figure S7: Transient Kinetic Fitting for Compound 5 in Methanol Solution & 17 \\
\hline 5. Global Analysis of Transient Absorption Data & 18 \\
\hline Figure S8: Global Analysis of TA Data for Compound 1 in Methanol Solution & 19 \\
\hline Figure S9: Global Analysis of TA Data for Compound 2 in Methanol Solution & 22 \\
\hline Figure S10: Global Analysis of TA Data for Compound 3 in Methanol Solution & 25 \\
\hline Figure S11: Global Analysis of TA Data for Compound 4 in Methanol Solution & 27 \\
\hline Figure S12: Global Analysis of TA Data for Compound 5 in Methanol Solution & 30 \\
\hline Table S13: Time Constants from Alternative Model & 31 \\
\hline 6. Statistical Comparison of Fit Quality & 32 \\
\hline 7. Fitting Results for All Models & 34 \\
\hline Table S14: Fitted Time Constants and Residuals & 35 \\
\hline Table S15: Other Fitted Parameters & 36 \\
\hline 8. TCSPC Fitting Results & 37 \\
\hline Figure S13: TCSPC Fits and Residuals & 38 \\
\hline
\end{tabular}




\section{Electronic Structure Calculation Results}

Table S1 shows the total self consistent field (SCF) energies resulting from electronic structure calcualtions performed on each compound at various charges and spin multiplicities, along with the binding energies derived from these values. This data was used to construct the energy level diagrams shown in Figure 5 of the article. The structure of each compound was optimized in the electronic ground state, with singlet spin multiplicity and neutral charge. Each structure was pre-optimized with the semi-empirical PM6 method, then re-optimized with density functional theory (DFT) using the CAM-B3LYP functional and the 6-31G(d,p) basis set. The effects of methanol solvent were included through the polarizable continuum model. The total SCF energy from the DFT optimization is listed as the neutral singlet energy. The optimized structure was then used for single point energy calculations with zero charge and triplet spin multiplicity, resulting in the neutral triplet energies reported in the table, and with +1 charge and doublet spin multiplicity, yielding the cation doublet energies. Binding energies were calcualted by comparing the cation doublet energies, $E_{C D}$, with the corresponding energies of the neutral singlet, $E_{S_{0}}$, and the neutral triplet, $E_{T_{1}}$, according to the equations below.

$$
\begin{aligned}
& e B E_{S_{0}}=E_{C D}-E_{S_{0}} \\
& e B E_{T_{1}}=E_{C D}-E_{T_{1}}
\end{aligned}
$$

Table S1: Energies from Electronic Structure Calculations at the Optimized $S_{0}$ Geometry

\begin{tabular}{|l|c|c|c|c|c|}
\hline Compound & $\mathbf{E}_{\mathbf{S}_{\mathbf{0}}}(\mathbf{e V})$ & $\mathbf{E}_{\mathbf{T}_{\mathbf{0}}}(\mathbf{e V})$ & $\mathbf{E}_{\mathbf{C D}}(\mathbf{e V})$ & $\left.\mathbf{e B E}_{\mathbf{S}_{\mathbf{0}}} \mathbf{( e V}\right)$ & $\mathbf{e B E}_{\mathbf{T}_{\mathbf{1}}}(\mathbf{e V})$ \\
\hline $\mathbf{1}$ & -21824.818 & -21821.890 & -21819.860 & 4.958 & 2.928 \\
\hline $\mathbf{2}$ & -17563.297 & -17559.937 & -17557.713 & 5.583 & 3.360 \\
\hline $\mathbf{3}$ & -17563.185 & -17560.150 & -17557.556 & 5.629 & 3.035 \\
\hline $\mathbf{4}$ & -25487.033 & -25484.113 & -25481.220 & 5.813 & 2.921 \\
\hline $\mathbf{5}$ & -25486.952 & -25483.987 & -25481.214 & 5.738 & 2.965 \\
\hline
\end{tabular}

Tables S2 - S6 list the results of excited state calculations performed at the optimized ground state, neutral, singlet geometry of each compound. Excited state calculations were perfored with time dependent 
density functional theory (TDDFT), using the same CAM-B3LYP functional and 6-31G(d,p) basis set that was employed for the geometry optimization. The polarizable continuum model was again used to include the effect of methanol solvent. The ten lowest-energy singlet excited states of each compound were calculated. Each table shows the predicted excitation wavelength and oscillator strength, which were used in creation of the energy level diagrams shown in Figure 5 of the article. The molecular orbital transition participating most strongly in each predicted excitation are also listed, along with the associated LCAO coefficient, the percentage contribution to the transition (equal to twice the square of the LCAO coefficient), and the HOMO/LUMO status of the two molecular orbitals involved. For excited states with multiple molecular orbital transitions contributing nearly equally, additional data on molecular orbitals has been listed.

Table S2: Excited State Calculation Results for Compound 1 at the Optimized $S_{0}$ Geometry

\begin{tabular}{|l|c|c|c|c|c|c|}
\hline $\begin{array}{c}\text { Excited } \\
\text { State }\end{array}$ & $\begin{array}{c}\text { Excitation } \\
\text { Wavelength } \\
(\mathbf{n m})\end{array}$ & $\begin{array}{c}\text { Oscillator } \\
\text { Strength } \\
(\mathbf{u n i t l e s s )}\end{array}$ & $\begin{array}{c}\text { Strongest } \\
\text { Transition }\end{array}$ & $\begin{array}{c}\text { LCAO } \\
\text { Coefficient } \\
\text { (unitless) }\end{array}$ & $\begin{array}{c}\text { Percentage } \\
\text { Contribution }\end{array}$ & $\begin{array}{c}\text { HOMO/LUMO } \\
\text { Status }\end{array}$ \\
\hline $\mathbf{1}$ & 310.6 & 0.4990 & $68 \rightarrow 69$ & 0.67632 & 91.5 & HOMO $\rightarrow$ LUMO \\
\hline $\mathbf{2}$ & 269.4 & 0.0246 & $67 \rightarrow 69$ & 0.50940 & 51.9 & HOMO-1 $\rightarrow$ LUMO \\
\hline & & & $68 \rightarrow 70$ & 0.44704 & 40.0 & HOMO $\rightarrow$ LUMO+1 \\
\hline $\mathbf{3}$ & 245.5 & 0.1675 & $67 \rightarrow 69$ & -0.46986 & 44.2 & HOMO-1 $\rightarrow$ LUMO \\
\hline & & & $68 \rightarrow 70$ & 0.48929 & 47.9 & HOMO $\rightarrow$ LUMO+1 \\
\hline $\mathbf{4}$ & 237.1 & 0.0001 & $65 \rightarrow 69$ & 0.63051 & 79.5 & HOMO-3 $\rightarrow$ LUMO \\
\hline $\mathbf{5}$ & 215.6 & 0.1063 & $68 \rightarrow 71$ & 0.60980 & 74.4 & HOMO $\rightarrow$ LUMO+2 \\
\hline $\mathbf{6}$ & 209.6 & 0.7705 & $67 \rightarrow 70$ & 0.58719 & 69.0 & HOMO-1 $\rightarrow$ LUMO+1 \\
\hline $\mathbf{7}$ & 196.2 & 0.3529 & $66 \rightarrow 69$ & 0.58303 & 68.0 & HOMO-2 $\rightarrow$ LUMO \\
\hline $\mathbf{8}$ & 187.7 & 0.1804 & $67 \rightarrow 71$ & 0.64537 & 83.3 & HOMO-1 $\rightarrow$ LUMO+2 \\
\hline $\mathbf{9}$ & 178.5 & 0.0007 & $65 \rightarrow 70$ & 0.57333 & 65.7 & HOMO-3 $\rightarrow$ LUMO+1 \\
\hline $\mathbf{1 0}$ & 175.0 & 0.0486 & $66 \rightarrow 70$ & 0.58366 & 68.1 & HOMO-2 $\rightarrow$ LUMO+1 \\
\hline
\end{tabular}


Table S3: Excited State Calculation Results for Compound 2 at the Optimized $S_{0}$ Geometry

\begin{tabular}{|l|c|c|c|c|c|c|}
\hline $\begin{array}{c}\text { Excited } \\
\text { State }\end{array}$ & $\begin{array}{c}\text { Excitation } \\
\text { Wavelength } \\
(\mathbf{n m})\end{array}$ & $\begin{array}{c}\text { Oscillator } \\
\text { Strength } \\
\text { (unitless) }\end{array}$ & $\begin{array}{c}\text { Strongest } \\
\text { Transition }\end{array}$ & $\begin{array}{c}\text { LCAO } \\
\text { Coefficient } \\
\text { unitless) }\end{array}$ & $\begin{array}{c}\text { Percentage } \\
\text { Contribution }\end{array}$ & $\begin{array}{c}\text { HOMO/LUMO } \\
\text { Status }\end{array}$ \\
\hline $\mathbf{1}$ & 286.3 & 0.2039 & $52 \rightarrow 53$ & 0.65844 & 86.7 & HOMO $\rightarrow$ LUMO \\
\hline $\mathbf{2}$ & 260.6 & 0.0037 & $51 \rightarrow 53$ & 0.48576 & 47.2 & HOMO-1 $\rightarrow$ LUMO \\
\hline & & & $52 \rightarrow 54$ & -0.46684 & 43.6 & HOMO $\rightarrow$ LUMO+1 \\
\hline $\mathbf{3}$ & 236.5 & 0.0000 & $50 \rightarrow 53$ & 0.63735 & 81.2 & HOMO-2 $\rightarrow$ LUMO \\
\hline $\mathbf{4}$ & 231.8 & 0.7532 & $52 \rightarrow 54$ & 0.44505 & 39.6 & HOMO $\rightarrow$ LUMO+1 \\
\hline & & & $51 \rightarrow 54$ & -0.36271 & 26.3 & HOMO-1 $\rightarrow$ LUMO+1 \\
\hline & & & $51 \rightarrow 53$ & 0.36238 & 26.3 & HOMO-1 $\rightarrow$ LUMO \\
\hline $\mathbf{5}$ & 225.7 & 0.2201 & $51 \rightarrow 54$ & 0.49004 & 48.0 & HOMO-1 $\rightarrow$ LUMO+1 \\
\hline & & & $51 \rightarrow 53$ & 0.32480 & 21.1 & HOMO-1 $\rightarrow$ LUMO \\
\hline $\mathbf{6}$ & 197.6 & 0.2401 & $52 \rightarrow 55$ & 0.57272 & 65.6 & HOMO $\rightarrow$ LUMO+2 \\
\hline $\mathbf{7}$ & 193.1 & 0.1640 & $49 \rightarrow 53$ & 0.59395 & 70.6 & HOMO-3 $\rightarrow$ LUMO \\
\hline $\mathbf{8}$ & 186.9 & 0.1910 & $49 \rightarrow 54$ & 0.52211 & 54.5 & HOMO-3 $\rightarrow$ LUMO+1 \\
\hline $\mathbf{9}$ & 185.2 & 0.0000 & $50 \rightarrow 54$ & 0.58701 & 68.9 & HOMO-2 $\rightarrow$ LUMO+1 \\
\hline $\mathbf{1 0}$ & 181.6 & 0.0548 & $51 \rightarrow 55$ & 0.62061 & 77.0 & HOMO-1 $\rightarrow$ LUMO+2 \\
\hline
\end{tabular}

Table S4: Excited State Calculation Results for Compound 3 at the Optimized $S_{0}$ Geometry

\begin{tabular}{|l|c|c|c|c|c|c|}
\hline $\begin{array}{c}\text { Excited } \\
\text { State }\end{array}$ & $\begin{array}{c}\text { Excitation } \\
\text { Wavelength } \\
(\mathbf{n m})\end{array}$ & $\begin{array}{c}\text { Oscillator } \\
\text { Strength } \\
(\text { unitless })\end{array}$ & $\begin{array}{c}\text { Strongest } \\
\text { Transition }\end{array}$ & $\begin{array}{c}\text { LCAO } \\
\text { Coefficient } \\
\text { unitless })\end{array}$ & $\begin{array}{c}\text { Percentage } \\
\text { Contribution }\end{array}$ & $\begin{array}{c}\text { HOMO/LUMO } \\
\text { Status }\end{array}$ \\
\hline $\mathbf{1}$ & 302.4 & 0.2091 & $52 \rightarrow 53$ & 0.67537 & 91.2 & HOMO $\rightarrow$ LUMO \\
\hline $\mathbf{2}$ & 279.3 & 0.2540 & $51 \rightarrow 53$ & 0.66396 & 88.2 & HOMO-1 $\rightarrow$ LUMO \\
\hline $\mathbf{3}$ & 239.4 & 0.0001 & $50 \rightarrow 53$ & 0.59589 & 71.0 & HOMO-2 $\rightarrow$ LUMO \\
\hline $\mathbf{4}$ & 221.4 & 0.1148 & $52 \rightarrow 54$ & 0.47292 & 44.7 & HOMO $\rightarrow$ LUMO+1 \\
\hline $\mathbf{5}$ & 212.9 & 0.2348 & $49 \rightarrow 53$ & 0.44643 & 39.9 & HOMO-3 $\rightarrow$ LUMO \\
\hline & & & $52 \rightarrow 54$ & -0.40198 & 32.3 & HOMO $\rightarrow$ LUMO+1 \\
\hline $\mathbf{6}$ & 208.5 & 0.0705 & $51 \rightarrow 54$ & 0.51669 & 53.4 & HOMO-1 $\rightarrow$ LUMO+1 \\
\hline $\mathbf{7}$ & 197.0 & 0.5966 & $52 \rightarrow 54$ & 0.40747 & 33.2 & HOMO $\rightarrow$ LUMO+1 \\
\hline & & & $51 \rightarrow 54$ & -0.34257 & 23.5 & HOMO-1 $\rightarrow$ LUMO+1 \\
\hline $\mathbf{8}$ & 195.2 & 0.3738 & $51 \rightarrow 55$ & 0.60409 & 73.0 & HOMO-1 $\rightarrow$ LUMO+2 \\
\hline $\mathbf{9}$ & 187.4 & 0.0000 & $50 \rightarrow 54$ & 0.51526 & 53.1 & HOMO-2 $\rightarrow$ LUMO+1 \\
\hline $\mathbf{1 0}$ & 177.5 & 0.1275 & $48 \rightarrow 53$ & 0.60108 & 72.3 & HOMO-4 $\rightarrow$ LUMO \\
\hline
\end{tabular}


Table S5: Excited State Calculation Results for Compound 4 at the Optimized $S_{0}$ Geometry

\begin{tabular}{|l|c|c|c|c|c|c|}
\hline $\begin{array}{c}\text { Excited } \\
\text { State }\end{array}$ & $\begin{array}{c}\text { Excitation } \\
\text { Wavelength } \\
(\mathbf{n m})\end{array}$ & $\begin{array}{c}\text { Oscillator } \\
\text { Strength } \\
\text { (unitless) }\end{array}$ & $\begin{array}{c}\text { Strongest } \\
\text { Transition }\end{array}$ & $\begin{array}{c}\text { LCAO } \\
\text { Coefficient } \\
\text { unitless) }\end{array}$ & $\begin{array}{c}\text { Percentage } \\
\text { Contribution }\end{array}$ & $\begin{array}{c}\text { HOMO/LUMO } \\
\text { Status }\end{array}$ \\
\hline $\mathbf{1}$ & 311.8 & 0.1935 & $79 \rightarrow 80$ & 0.67131 & 90.1 & HOMO $\rightarrow$ LUMO \\
\hline $\mathbf{2}$ & 274.5 & 0.0819 & $78 \rightarrow 80$ & 0.56046 & 62.8 & HOMO-1 $\rightarrow$ LUMO \\
\hline $\mathbf{3}$ & 243.5 & 0.0842 & $74 \rightarrow 80$ & 0.38865 & 30.2 & HOMO-5 $\rightarrow$ LUMO \\
\hline $\mathbf{4}$ & 241.7 & 0.1949 & $79 \rightarrow 81$ & 0.37329 & 27.9 & HOMO $\rightarrow$ LUMO+1 \\
\hline $\mathbf{5}$ & 233.7 & 0.8064 & $79 \rightarrow 81$ & 0.41050 & 33.7 & HOMO $\rightarrow$ LUMO+1 \\
\hline & & & $78 \rightarrow 80$ & 0.38727 & 30.0 & HOMO-1 $\rightarrow$ LUMO \\
\hline $\mathbf{6}$ & 222.9 & 0.0003 & $77 \rightarrow 83$ & 0.40576 & 32.9 & HOMO-2 $\rightarrow$ LUMO+3 \\
\hline $\mathbf{7}$ & 214.6 & 0.1779 & $78 \rightarrow 81$ & 0.50182 & 50.4 & HOMO-1 $\rightarrow$ LUMO+1 \\
\hline $\mathbf{8}$ & 213.3 & 0.4180 & $75 \rightarrow 80$ & 0.45348 & 41.1 & HOMO-4 $\rightarrow$ LUMO \\
\hline $\mathbf{9}$ & 211.1 & 0.0860 & $77 \rightarrow 80$ & 0.57605 & 66.4 & HOMO-2 $\rightarrow$ LUMO \\
\hline $\mathbf{1 0}$ & 201.3 & 0.0292 & $79 \rightarrow 83$ & 0.33803 & 22.9 & HOMO $\rightarrow$ LUMO +3 \\
\hline & & & $79 \rightarrow 84$ & -0.31044 & 19.3 & HOMO $\rightarrow$ LUMO +4 \\
\hline
\end{tabular}

Table S6: Excited State Calculation Results for Compound 5 at the Optimized $S_{0}$ Geometry

\begin{tabular}{|l|c|c|c|c|c|c|}
\hline $\begin{array}{c}\text { Excited } \\
\text { State }\end{array}$ & $\begin{array}{c}\text { Excitation } \\
\text { Wavelength } \\
(\mathbf{n m})\end{array}$ & $\begin{array}{c}\text { Oscillator } \\
\text { Strength } \\
\text { (unitless) }\end{array}$ & $\begin{array}{c}\text { Strongest } \\
\text { Transition }\end{array}$ & $\begin{array}{c}\text { LCAO } \\
\text { Coefficient } \\
\text { unitless) }\end{array}$ & $\begin{array}{c}\text { Percentage } \\
\text { Contribution }\end{array}$ & $\begin{array}{c}\text { HOMO/LUMO } \\
\text { Status }\end{array}$ \\
\hline $\mathbf{1}$ & 311.6 & 0.1374 & $79 \rightarrow 80$ & 0.66763 & 89.1 & HOMO $\rightarrow$ LUMO \\
\hline $\mathbf{2}$ & 281.9 & 0.0593 & $79 \rightarrow 81$ & 0.58589 & 68.7 & HOMO $\rightarrow$ LUMO+1 \\
\hline $\mathbf{3}$ & 250.1 & 0.6489 & $78 \rightarrow 80$ & 0.45669 & 41.7 & HOMO-1 $\rightarrow$ LUMO \\
\hline $\mathbf{4}$ & 243.3 & 0.1253 & $74 \rightarrow 80$ & 0.43552 & 37.9 & HOMO-5 $\rightarrow$ LUMO \\
\hline $\mathbf{5}$ & 226.0 & 0.2431 & $78 \rightarrow 81$ & 0.38567 & 29.7 & HOMO-1 $\rightarrow$ LUMO+1 \\
\hline & & & $78 \rightarrow 80$ & 0.35177 & 24.7 & HOMO-1 $\rightarrow$ LUMO \\
\hline $\mathbf{6}$ & 223.2 & 0.0091 & $77 \rightarrow 82$ & 0.34980 & 24.5 & HOMO-2 $\rightarrow$ LUMO+2 \\
\hline $\mathbf{7}$ & 215.5 & 0.2737 & $75 \rightarrow 80$ & 0.39349 & 31.0 & HOMO-4 $\rightarrow$ LUMO \\
\hline & & & $78 \rightarrow 84$ & 0.35681 & 25.5 & HOMO-1 $\rightarrow$ LUMO +4 \\
\hline $\mathbf{8}$ & 211.5 & 0.0691 & $79 \rightarrow 84$ & 0.34229 & 23.4 & HOMO $\rightarrow$ LUMO+4 \\
\hline $\mathbf{9}$ & 208.3 & 0.2330 & $77 \rightarrow 80$ & 0.55547 & 61.7 & HOMO-2 $\rightarrow$ LUMO \\
\hline $\mathbf{1 0}$ & 204.1 & 0.0162 & $79 \rightarrow 82$ & 0.53664 & 57.6 & HOMO $\rightarrow$ LUMO+2 \\
\hline
\end{tabular}


Tables S7 - S11 show the results of excited state calculations performed at the optimized $\mathrm{S}_{1}$ geometry of each neutrally charged compound. The excited state geometry optimization was performed starting from the previsouly-optimized, ground state, neutral structure. TDDFT calcualtions were carried out using the CAM-B3LYP functional and the 6-31G(d,p) basis set, including the methanol solvent via the polarizable continuum model. The ten lowest energy singlet excited states of each compound were calculated. Predicted $\mathrm{S}_{1} \rightarrow \mathrm{S}_{\mathrm{n}}$ transition energies were calculated as the difference between the predicted transition energy of the $S_{1}$ state and the the predicted transition energy for each higher lying $S_{n}$ state. These energy values were then converted to corresponding photon wavelengths, for the purpose of assigning excited state absorption features in the transient absortpion spectra. Each table reports the predicted oscillator strength and transition energy for each the $S_{0} \rightarrow S_{n}$ transition, along with the predicted $S_{1} \rightarrow S_{n}$ transition energies and corresponding wavelengths.

Table S7: Excited State Calculation Results for Compound 1 at the Optimized $S_{1}$ Geometry

\begin{tabular}{|c|c|c|c|c|}
\hline $\begin{array}{l}\text { Excited } \\
\text { State }\end{array}$ & $\begin{array}{l}S_{0} \rightarrow S_{n} \text { Oscillator } \\
\text { Strength (unitless) }\end{array}$ & $\begin{array}{c}S_{0} \rightarrow S_{n} \text { Transition } \\
\text { Energy }(\mathrm{eV})\end{array}$ & $\begin{array}{c}S_{1} \rightarrow S_{n} \text { Transition } \\
\text { Energy }(e V)\end{array}$ & $\begin{array}{l}S_{1} \rightarrow S_{n} \text { Transition } \\
\text { Wavelength }(\mathrm{nm})\end{array}$ \\
\hline 1 & 0.5554 & 3.6591 & 0.0000 & $x$ \\
\hline 2 & 0.0060 & 4.3705 & 0.7114 & 1743 \\
\hline 3 & 0.1402 & 4.9307 & 1.2716 & 975.02 \\
\hline 4 & 0.0020 & 5.0297 & 1.3706 & 904.60 \\
\hline 5 & 0.1872 & 5.6095 & 1.9504 & 635.68 \\
\hline 6 & 0.3329 & 5.8153 & 2.1562 & 575.01 \\
\hline 7 & 0.7573 & 6.0842 & 2.4251 & 511.25 \\
\hline 8 & 0.1302 & 6.5466 & 2.8875 & 429.38 \\
\hline 9 & 0.0596 & 6.8434 & 3.1843 & 389.36 \\
\hline 10 & 0.0014 & 6.8781 & 3.2190 & 385.16 \\
\hline
\end{tabular}


Table S8: Excited State Calculation Results for Compound 2 at the Optimized $S_{1}$ Geometry

\begin{tabular}{|l|c|c|c|c|}
\hline $\begin{array}{c}\text { Excited } \\
\text { State }\end{array}$ & $\begin{array}{c}\text { So }_{\mathbf{0}} \rightarrow \mathbf{S}_{\mathbf{n}} \text { Oscillator } \\
\text { Strength } \\
\text { (unitless) }\end{array}$ & $\begin{array}{c}\text { S0 to Sn } \\
\text { Transition } \\
\text { Energy (eV) }\end{array}$ & $\begin{array}{c}\text { S1 to Sn } \\
\text { Transition } \\
\text { Energy (eV) }\end{array}$ & $\begin{array}{c}\text { S1 to Sn } \\
\text { Transition } \\
\text { Wavelength } \\
\text { (nm) }\end{array}$ \\
\hline 1 & 0.2173 & 3.7645 & 0.0000 & x \\
\hline 2 & 0.0275 & 4.4179 & 0.6534 & 1898 \\
\hline 3 & 0.0000 & 4.9714 & 1.2069 & 1027.3 \\
\hline 4 & 0.7042 & 5.0551 & 1.2906 & 960.67 \\
\hline 5 & 0.1473 & 5.4062 & 1.6417 & 755.22 \\
\hline 6 & 0.2161 & 6.0131 & 2.2486 & 551.38 \\
\hline 7 & 0.0985 & 6.0764 & 2.3119 & 536.29 \\
\hline 8 & 0.5206 & 6.4456 & 2.6811 & 462.44 \\
\hline 9 & 0.0000 & 6.6096 & 2.8451 & 435.78 \\
\hline 10 & 0.2253 & 6.6441 & 2.8796 & 430.56 \\
\hline
\end{tabular}

Table S9: Excited State Calculation Results for Compound 3 at the Optimized $S_{1}$ Geometry

\begin{tabular}{|l|c|c|c|c|}
\hline $\begin{array}{c}\text { Excited } \\
\text { State }\end{array}$ & $\begin{array}{c}S_{0} \rightarrow S_{n} \text { Oscillator } \\
\text { Strength (unitless) }\end{array}$ & $\begin{array}{c}S_{0} \rightarrow S_{n} \text { Transition } \\
\text { Energy }(\mathbf{e V})\end{array}$ & $\begin{array}{c}S_{1} \rightarrow S_{n} \text { Transition } \\
\text { Energy }(\mathbf{e V})\end{array}$ & $\begin{array}{c}\boldsymbol{S}_{1} \rightarrow \mathbf{S}_{\mathbf{n}} \text { Transition } \\
\text { Wavelength }(\mathbf{n m})\end{array}$ \\
\hline 1 & 0.1915 & 3.6101 & 0.0000 & $\mathbf{x}$ \\
\hline 2 & 0.2847 & 4.1604 & 0.5503 & 2253 \\
\hline 3 & 0.0001 & 4.9105 & 1.3004 & 953.43 \\
\hline 4 & 0.1216 & 5.3668 & 1.7567 & 705.78 \\
\hline 5 & 0.2031 & 5.5200 & 1.9099 & 649.16 \\
\hline 6 & 0.1919 & 5.8084 & 2.1983 & 564.00 \\
\hline 7 & 0.4748 & 6.1216 & 2.5115 & 493.66 \\
\hline 8 & 0.2885 & 6.4001 & 2.7900 & 444.39 \\
\hline 9 & 0.0000 & 6.4740 & 2.8639 & 432.92 \\
\hline 10 & 0.1903 & 6.6708 & 3.0607 & 405.08 \\
\hline
\end{tabular}


Table S10: Excited State Calculation Results for Compound 4 at the Optimized $S_{1}$ Geometry

\begin{tabular}{|l|r|c|c|c|}
\hline $\begin{array}{c}\text { Excited } \\
\text { State }\end{array}$ & $\begin{array}{c}\mathbf{S}_{0} \rightarrow \mathbf{S}_{\mathbf{n}} \text { Oscillator } \\
\text { Strength (unitless) }\end{array}$ & $\begin{array}{c}\mathbf{S}_{0} \rightarrow \mathbf{S}_{\mathbf{n}} \text { Transition } \\
\text { Energy (eV) }\end{array}$ & $\begin{array}{c}\mathbf{S}_{1} \rightarrow \mathbf{S}_{\mathbf{n}} \text { Transition } \\
\text { Energy (eV) }\end{array}$ & $\begin{array}{c}\mathbf{S}_{1} \rightarrow \mathbf{S}_{\mathbf{n}} \text { Transition } \\
\text { Wavelength (nm) }\end{array}$ \\
\hline 1 & 0.2255 & 3.3190 & 0.0000 & $\mathbf{x}$ \\
\hline 2 & 0.1339 & 4.0673 & 0.7483 & 1657 \\
\hline 3 & 0.0491 & 4.7270 & 1.4080 & 880.57 \\
\hline 4 & 0.4000 & 4.8526 & 1.5336 & 808.45 \\
\hline 5 & 0.2210 & 4.9253 & 1.6063 & 771.86 \\
\hline 6 & 0.2362 & 5.2908 & 1.9718 & 628.79 \\
\hline 7 & 0.0290 & 5.3910 & 2.0720 & 598.38 \\
\hline 8 & 0.0006 & 5.5511 & 2.2321 & 555.46 \\
\hline 9 & 0.4245 & 5.6444 & 2.3254 & 533.17 \\
\hline 10 & 0.0031 & 5.6906 & 2.3716 & 522.79 \\
\hline
\end{tabular}

Table S11: Excited State Calculation Results for Compound 5 at the Optimized $S_{1}$ Geometry

\begin{tabular}{|l|c|c|c|c|}
\hline $\begin{array}{c}\text { Excited } \\
\text { State }\end{array}$ & $\begin{array}{c}\mathbf{S}_{\mathbf{0}} \rightarrow \mathbf{S}_{\mathbf{n}} \text { Oscillator } \\
\text { Strength (unitless) }\end{array}$ & $\begin{array}{c}\mathbf{S}_{\mathbf{0}} \rightarrow \mathbf{S}_{\mathbf{n}} \text { Transition } \\
\text { Energy }(\mathbf{e V})\end{array}$ & $\begin{array}{c}\mathbf{S}_{\mathbf{1}} \rightarrow \mathbf{S}_{\mathbf{n}} \text { Transition } \\
\text { Energy }(\mathbf{e V})\end{array}$ & $\begin{array}{c}\mathbf{S}_{\mathbf{1}} \rightarrow \mathbf{S}_{\mathbf{n}} \text { Transition } \\
\text { Wavelength }(\mathbf{n m})\end{array}$ \\
\hline 1 & 0.1637 & 3.3789 & 0.0000 & $\mathbf{x}$ \\
\hline 2 & 0.0256 & 4.0898 & 0.7109 & 1744 \\
\hline 3 & 0.6396 & 4.6200 & 1.2411 & 998.98 \\
\hline 4 & 0.1493 & 4.8070 & 1.4281 & 868.17 \\
\hline 5 & 0.1389 & 5.1751 & 1.7962 & 690.26 \\
\hline 6 & 0.1885 & 5.4343 & 2.0554 & 603.21 \\
\hline 7 & 0.0203 & 5.4652 & 2.0863 & 594.28 \\
\hline 8 & 0.0015 & 5.5510 & 2.1721 & 570.80 \\
\hline 9 & 0.3182 & 5.6786 & 2.2997 & 539.13 \\
\hline 10 & 0.0006 & 5.8664 & 2.4875 & 498.43 \\
\hline
\end{tabular}




\section{Vibronic Peak Progressions Observed in Some Absorbance Spectra}

Figure 4 in the article shows that the absorbance spectra of molecules $\mathbf{4}$ and $\mathbf{5}$ include a vibronic progression in the lowest energy feature, with an energy spacing of $\sim 1250 \mathrm{~cm}^{-1}$ between the vibronic peaks. A similar progression may also be present for compounds $\mathbf{2}$ and $\mathbf{3}$, although the vibronic peaks are not resolved in this case. Computational results include predictions of the vibrational modes of the molecules, including both vibrational frequencies and associated atomic motions. While an exact assignment of the observed vibronic modes is not possible, the modes with vibrational frequencies most closely matching to the observed energy spacing of the vibronic peaks can be noted, and their associated atomic motions can be qualitatively characterized.

The vibronic peaks are most clearly observed in compounds $\mathbf{4}$ and $\mathbf{5}$. For compound $\mathbf{4}$, clear maxima were observed at $353( \pm 1) \mathrm{nm}$ and $369( \pm 1) \mathrm{nm}$, with an unresolved shoulder near 339 $\mathrm{nm}$. The reported uncertainty corresponds to the data point spacing in the measurement, which is much larger than the resolution of the spectrometer, but an appropriately safe uncertainty estimate for this room temperature, solution phase measurement. The corresponding energy gap between the two well-resolved peaks is $\sim 1228( \pm 100) \mathrm{cm}^{-1}$. For compound 5, well-resolved peaks were observed at $358( \pm 1) \mathrm{nm}$ and $375( \pm 1) \mathrm{nm}$, with a shoulder near $342( \pm 1) \mathrm{nm}$, and the corresponding energy spacing was $1265( \pm 100) \mathrm{cm}^{-1}$. For compounds $\mathbf{2}$ and $\mathbf{3}$, the peaks are not well resolved enough to assign peak wavelengths, but the spacing of the unresolved features appears to be of similar magnitude to that observed in compounds $\mathbf{4}$ and $\mathbf{5}$. The absorbance spectrum of compound 1 does not show any indication of vibronic structure.

Table S12 below lists the vibrational modes of compounds $\mathbf{2}-\mathbf{5}$ that appear in the range $1150 \mathrm{~cm}^{-1}$ to $1350 \mathrm{~cm}^{-1}$, along with their predicted relative strength in infrared absorbance spectra. 
All results reported are from DFT frequency calculations performed on the optimized singlet ground state structures, using the CAM-B3LYP functional and the 6-31G(d,p) basis set and including the methanol solvent via the polarizable continuum model. Inspection of the motions associated with these modes shows that they can all be characterized as collective ring motions, consisting of relative motion of the atoms making up the ring while preserving the planarity of the ring. The patterns of motion and portions of the ring structure that participate most strongly are different for each vibrational mode in each molecule. No out-of-plane motions, such as twisting or bending of the ring or out-of-plane waggling of terminal hydrogen atoms, were observed in any mode. Some modes observed in compounds $\mathbf{4}$ and $\mathbf{5}$ include motions localized on the pendant phenyl groups of those molecules, which also do not involve ring twisting or bending. The motions of the mode of compounds $\mathbf{4}$ and $\mathbf{5}$ that each most closely match the observed peak spacings in the respective absorbance spectra are shown below in Figure S1.
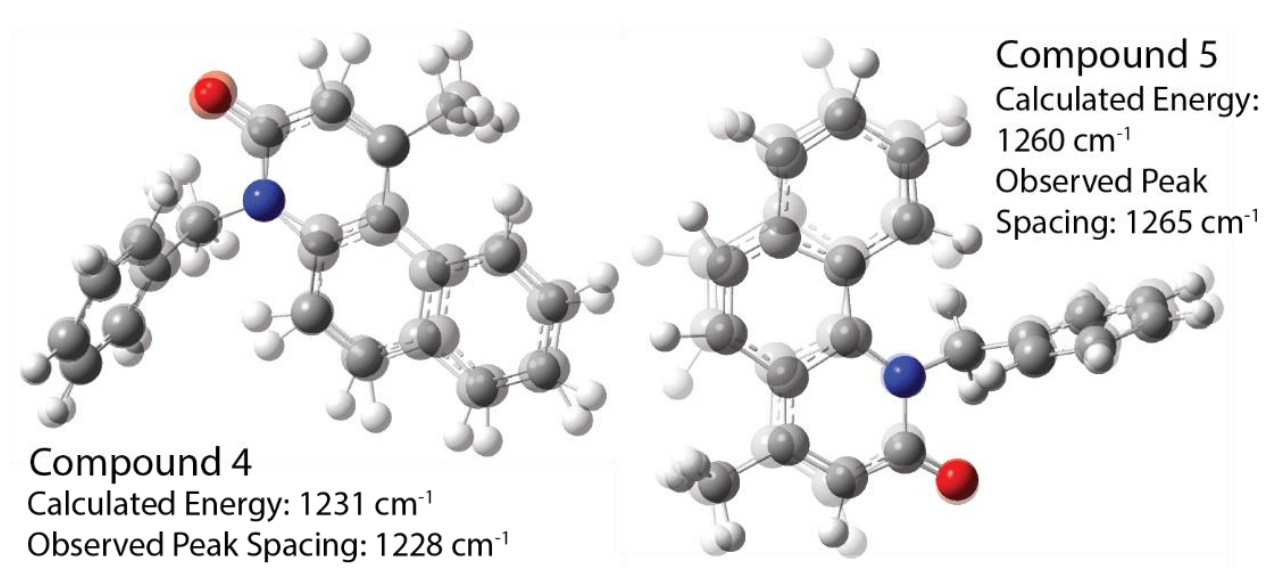

Figure S1: Motion along normal coordinates for compounds 4 and 5 . For $\mathbf{4}$ and $\mathbf{5}$, the atomic positions at the two extremes of vibrational motion are overlayed as partially transparent images. The images have been aligned on their nitrogen atoms by eye, to facilitate visualization of the relative motions of the other atoms. The vibrational modes shown in this figure are those with energies most closely matching the observed vibronic peak energy spacings for each compound. The relevant energetics are summarized in the figure. The atoms in the structures are color coded as: $\mathrm{C}$ (gray), $\mathrm{H}$ (white), $\mathrm{N}$ (blue), $\mathrm{O}$ (red). 
Table S12: Predicted Vibrational Modes With Frequencies Near the Observed Vibronic Energy Spacing

\begin{tabular}{|c|c|c|}
\hline Compound & Peak Energy $\left(\mathrm{cm}^{-1}\right)$ & Relative Infrared Absorbance Intensity \\
\hline 2 & 1163.68 & 5.1967 \\
\hline 2 & 1178.12 & 1.9414 \\
\hline 2 & 1223.51 & 10.9732 \\
\hline 2 & 1252.58 & 3.6335 \\
\hline 2 & 1281.59 & 1.9864 \\
\hline 2 & 1306.54 & 25.9270 \\
\hline 2 & 1341.83 & 22.6611 \\
\hline 3 & 1174.95 & 6.5827 \\
\hline 3 & 1212.59 & 30.9620 \\
\hline 3 & 1254.27 & 37.6423 \\
\hline 3 & 1281.77 & 6.2435 \\
\hline 3 & 1305.01 & 40.6048 \\
\hline 3 & 1348.57 & 26.0168 \\
\hline 4 & 1152.25 & 12.9995 \\
\hline 4 & 1178.57 & 7.1272 \\
\hline 4 & 1187.06 & 0.3000 \\
\hline 4 & 1193.35 & 18.2733 \\
\hline 4 & 1213.87 & 3.0933 \\
\hline 4 & 1220.26 & 6.4622 \\
\hline 4 & 1230.81 & 3.8368 \\
\hline 4 & 1249.19 & 0.4095 \\
\hline 4 & 1260.97 & 9.0697 \\
\hline 4 & 1271.52 & 2.2345 \\
\hline 4 & 1306.21 & 6.1002 \\
\hline 4 & 1335.68 & 5.5983 \\
\hline 5 & 1176.23 & 5.3745 \\
\hline 5 & 1186.20 & 0.2763 \\
\hline 5 & 1200.68 & 1.6606 \\
\hline 5 & 1209.13 & 14.8691 \\
\hline 5 & 1211.91 & 1.2296 \\
\hline 5 & 1225.30 & 19.0175 \\
\hline 5 & 1239.61 & 0.6434 \\
\hline 5 & 1257.07 & 5.0981 \\
\hline 5 & 1259.87 & 2.2226 \\
\hline 5 & 1306.04 & 9.4774 \\
\hline 5 & 1334.36 & 6.0279 \\
\hline
\end{tabular}




\section{Transient Absorbance Spectra in Methanol Solution}
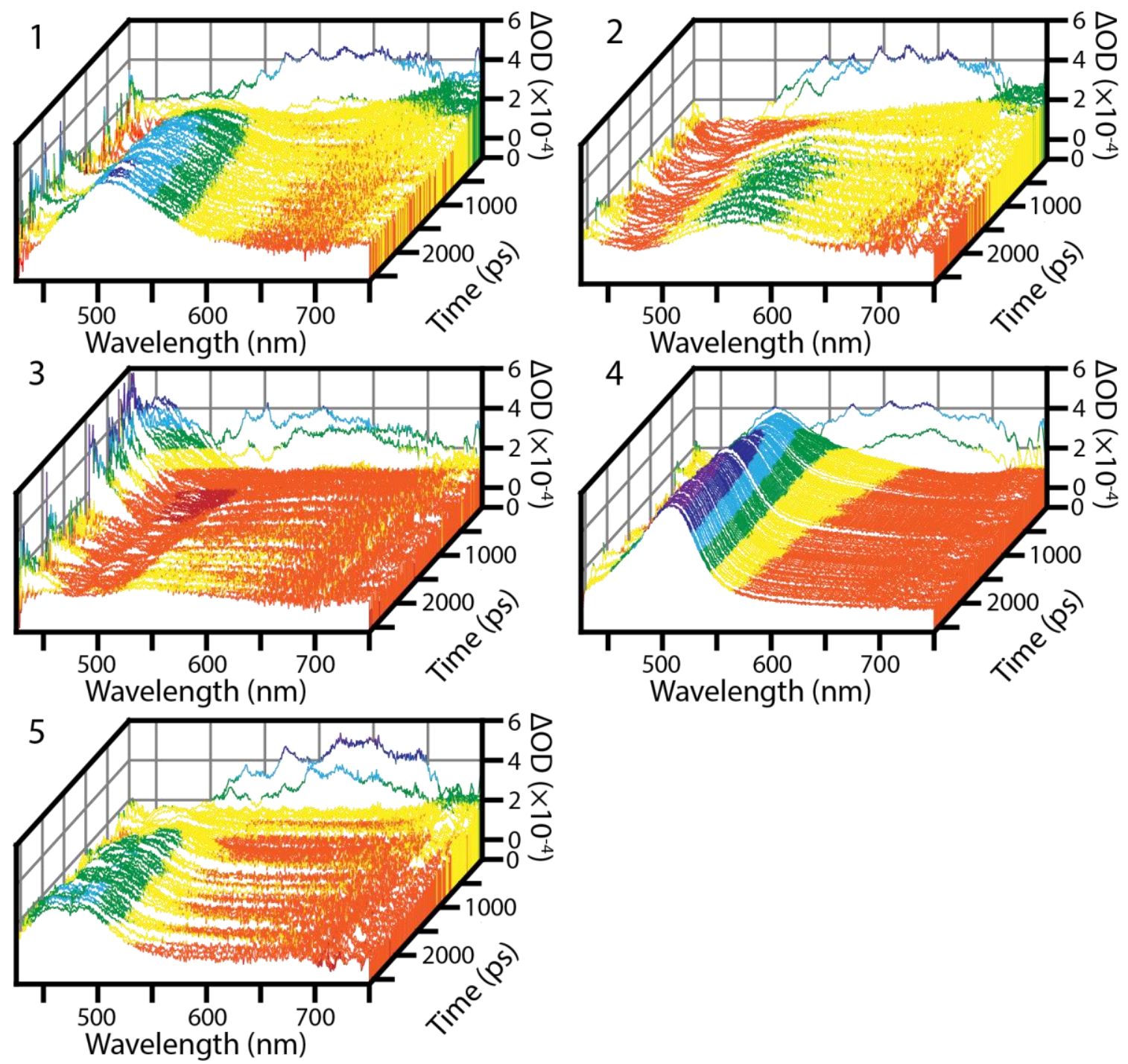

Figure S2: Waterfall plots of transient absorbance data for all compounds. Transient absorbance data sets for each compound $\mathbf{1}$ through $\mathbf{5}$ are shown as waterfall plots, displaying the signal magnitude as a function of both wavelength time delay. All data sets are displayed along a common set of identical axes, to facilitate comparison. A common color scale is used to highlight the changes in $\triangle \mathrm{OD}$ (all values $\times 10^{-4}$ ): -1.0 to 0 (red), 0 to 1.0 (orange), 1.0 to 2.0 (yellow), 2.0 to 3.0 (green), 3.0 to 4.0 (cyan), 4.0 to 5.0 (blue), and 5.0 to 6.0 (purple).

Results from transient absorption (TA) spectroscopy of compounds $\mathbf{1}$ through $\mathbf{5}$ are shown in

Figure S2. Spectra were acquired at room temperature in methanol solution, using an excitation wavelength of $350 \mathrm{~nm}$ and $400 \mu \mathrm{W}$ of excitation power at a repetition rate of $2.5 \mathrm{kHz}$. TA spectra 
of $\mathbf{2}$ and $\mathbf{4}$ are also shown in the article text, in Figures 8 and 9 respectively. Here all spectra are shown for completeness, and are placed on identically scaled axes and with a common color scale to facilitate comparison.

\section{Fitting Kinetic Traces of TA Data}

As a first method of characterizing the dynamics in the TA data set, kinetic traces were generated at selected wavelengths and fit to a multiexponential model. The results are shown below in Figures S3 - S7. Each data set generated for fitting is the result of averaging data recorded in a $4 \mathrm{~nm}$ window, centered at the specified value. The two wavelengths selected for each data set were the peak wavelength of the long lived feature, chosen to represent dynamics of the triplet population, and a region at long wavelengths, chosen to represent dynamics of the $S_{1}$ excited state. As described in the article, TA data at these wavelengths did not perfect represent the population dynamics of the associated states due to overlap of multiple spectral features. This is especially true of the data for the long-lived feature, which overlaps strongly with stimulated emission (SE) signal from the $\mathrm{S}_{1}$ state. Still, the fits reported below provide a sense of how the TA signal evolved with time in the most critical spectral regions. Data acquired at time delays less than 3 ps was excluded from fitting to avoid distortion of the fitted time constants by large numbers of data points recorded at short time delays and the high amplitude coherent artifacts present at early times in all data sets. Fit equations are reported in the figure captions, with the reported uncertainties being standard errors for the fit parameters. 

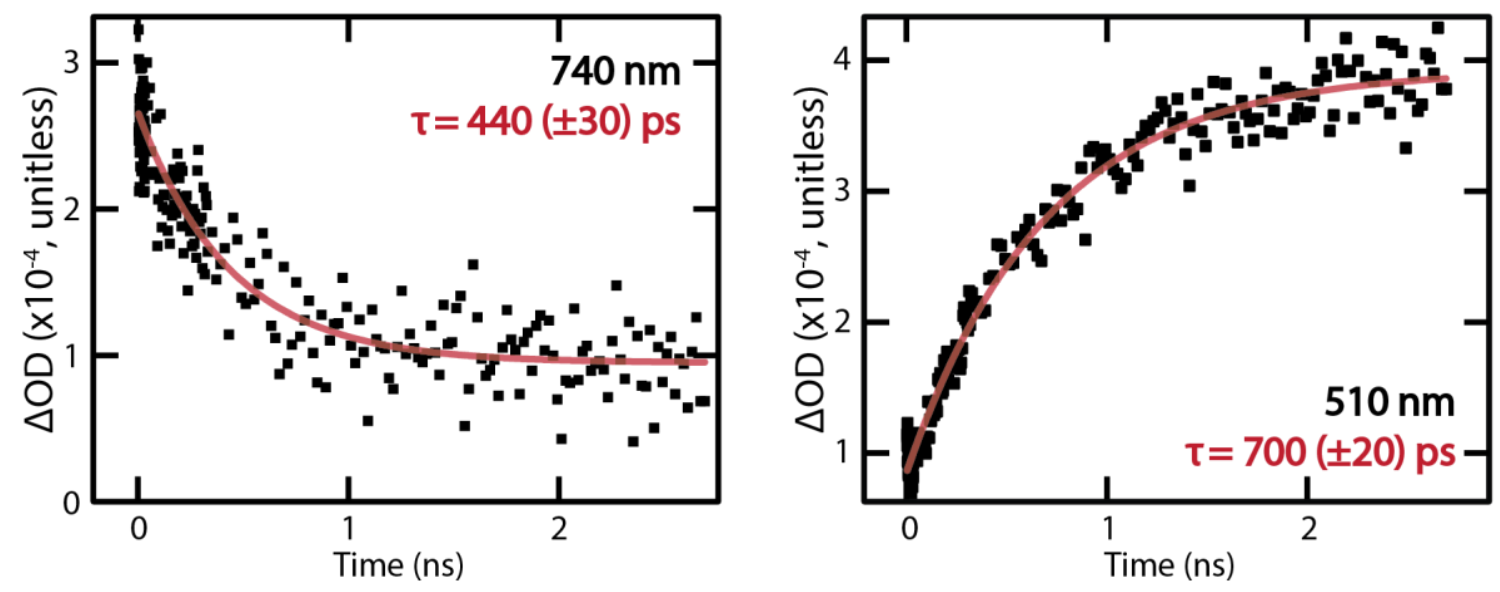

Figure S3: Transient Kinetic Fitting for Compound 1 in Methanol Solution. [left] Transient absorbance signal acquired at $740( \pm 2) \mathrm{nm}$, along with an exponential decay fit resulting in the fit equation $\triangle O D=4.1( \pm 0.9) \times 10^{-5}+(1.71( \pm 0.06) \times$ $\left.10^{-4}\right) \exp \left(-\frac{t}{440( \pm 30) p s}\right)$. [right] Transient absorbance signal acquired at $510( \pm 2) \mathrm{nm}$, along with an exponential decay fit resulting in the fit equation $\triangle O D=3.93( \pm 0.03) \times$ $10^{-4}+\left(-3.07( \pm 0.03) \times 10^{-4}\right) \exp \left(\frac{t}{700( \pm 20) p s}\right)$.
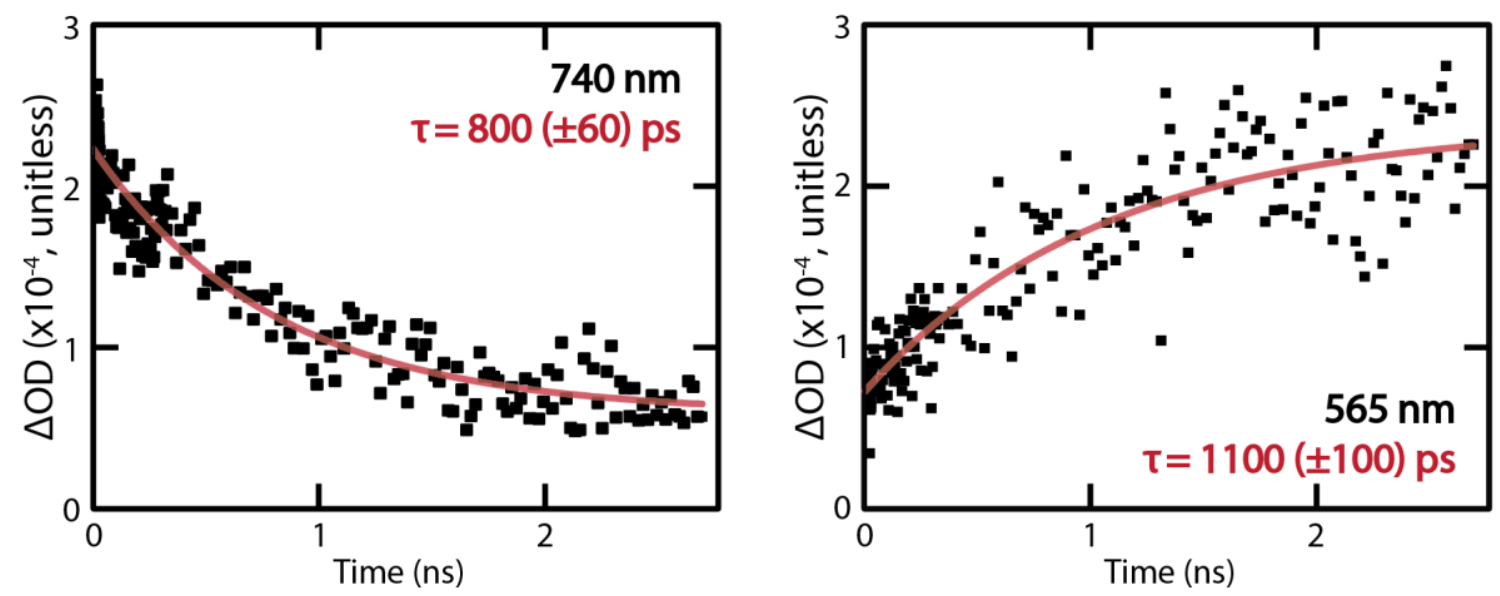

Figure S4: Transient Kinetic Fitting for Compound 2 in Methanol Solution. [left] Transient absorbance signal acquired at $740( \pm 2) \mathrm{nm}$, along with an exponential decay fit resulting in the fit equation $\triangle O D=5.9( \pm 0.4) \times 10^{-5}+(1.65( \pm 0.04) \times$ $\left.10^{-4}\right) \exp \left(-\frac{t}{800( \pm 60) p s}\right)$. [right] Transient absorbance signal acquired at $565( \pm 2) \mathrm{nm}$, along with an exponential decay fit resulting in the fit equation $\triangle O D=2.38( \pm 0.09) \times$ $10^{-4}+\left(-1.66( \pm 0.08) \times 10^{-4}\right) \exp \left(\frac{t}{1100( \pm 100) p s}\right)$. 

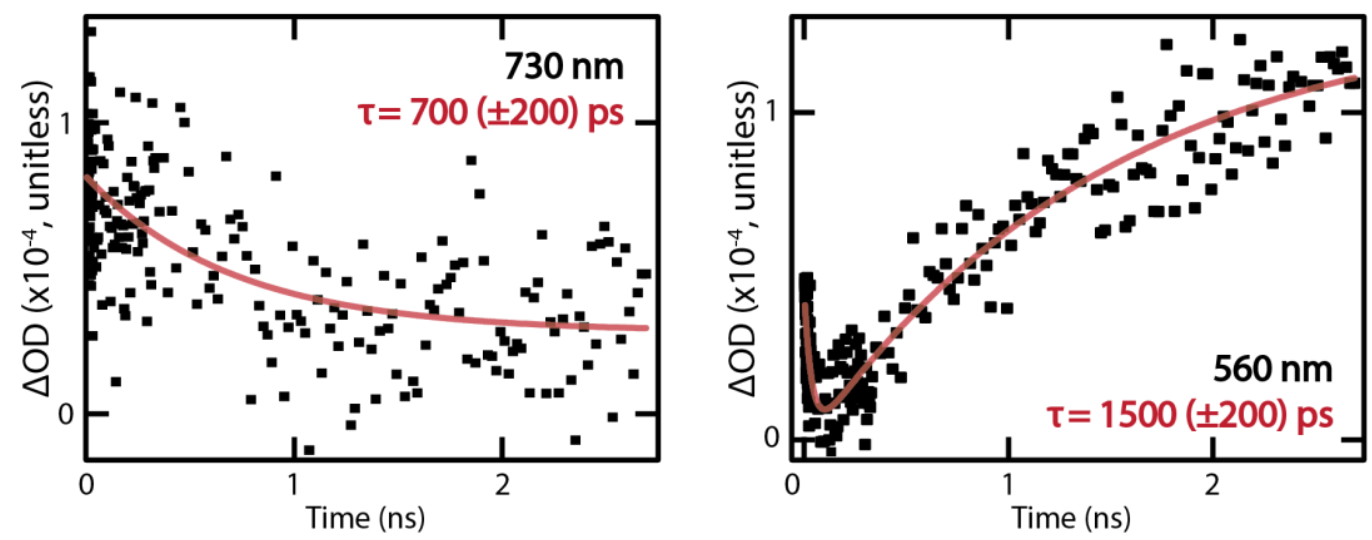

Figure S5: Transient Kinetic Fitting for Compound 3 in Methanol Solution. [left] Transient absorbance signal acquired at $730( \pm 2) \mathrm{nm}$, along with an exponential decay fit resulting in the fit equation $\triangle O D=2.8( \pm 0.4) \times 10^{-5}+(5.3( \pm 0.4) \times$ $\left.10^{-4}\right) \exp \left(-\frac{t}{700( \pm 200) p s}\right)$. [right] Transient absorbance signal acquired at $560( \pm 2) \mathrm{nm}$, along with a bi-exponential fit resulting in the fit equation $\triangle O D=1.32( \pm 0.08) \times 10^{-4}+$ $\left(-1.35( \pm 0.07) \times 10^{-4}\right) \exp \left(\frac{t}{1500( \pm 200) p s}\right)+\left(4.8( \pm 0.4) \times 10^{-5}\right)$

$\exp \left(-\frac{t}{37( \pm 8) p s}\right)$.
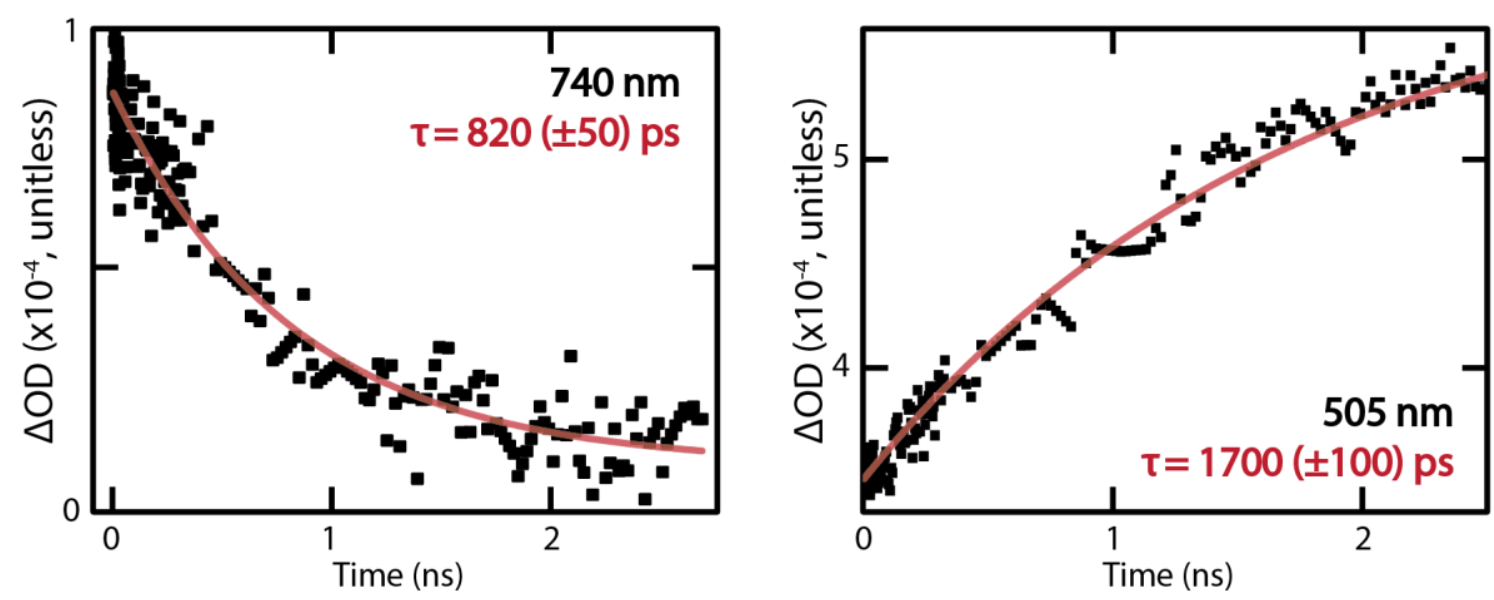

Figure S6: Transient Kinetic Fitting for Compound 4 in Methanol Solution. [left] Transient absorbance signal acquired at $740( \pm 2) \mathrm{nm}$, along with an exponential decay fit resulting in the fit equation $\triangle O D=8( \pm 2) \times 10^{-6}+(7.8( \pm 0.2) \times$ $\left.10^{-5}\right) \exp \left(-\frac{t}{820( \pm 50) p s}\right)$. [right] Transient absorbance signal acquired at $505( \pm 2) \mathrm{nm}$, along with an exponential decay fit resulting in the fit equation $\triangle O D=5.99( \pm 0.08) \times$ $10^{-4}+\left(-2.52( \pm 0.07) \times 10^{-4}\right) \exp \left(\frac{t}{1700( \pm 100) p s}\right)$. 

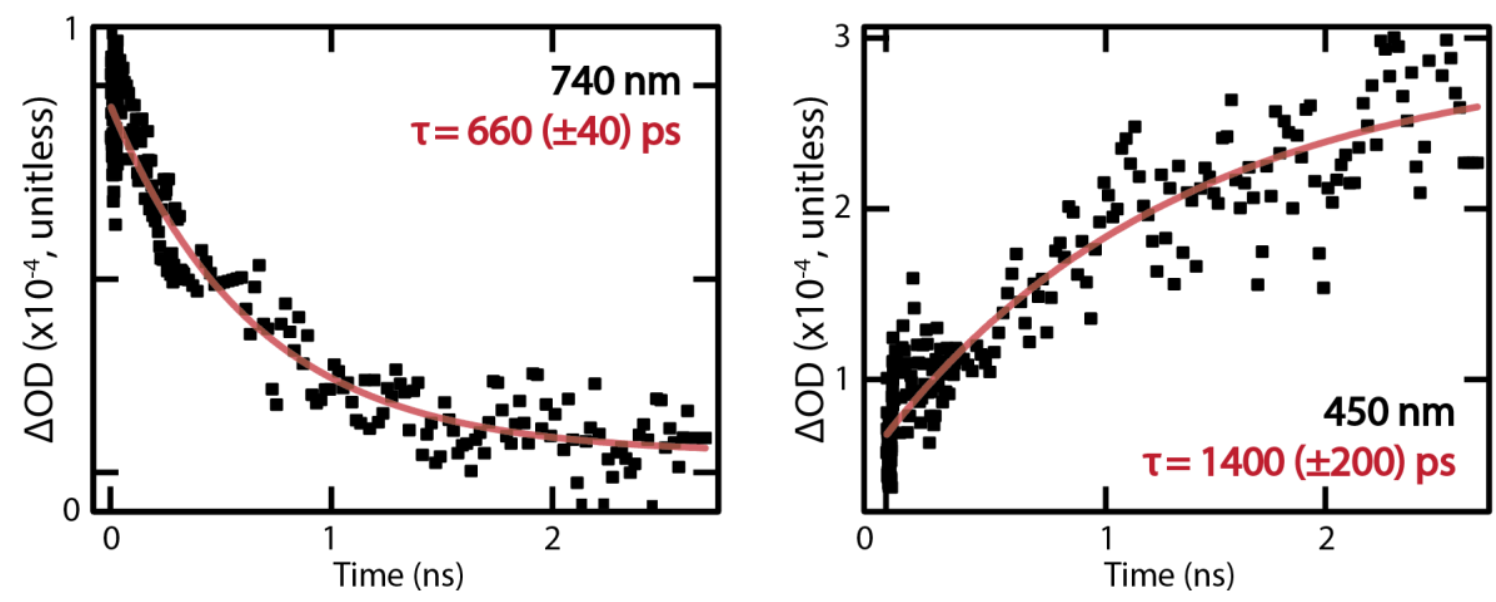

Figure S7: Transient Kinetic Fitting for Compound 5 in Methanol Solution. [left] Transient absorbance signal acquired at $740( \pm 2) \mathrm{nm}$, along with an exponential decay fit resulting in the fit equation $\triangle O D=1.0( \pm 0.3) \times 10^{-5}+(1.80( \pm 0.04) \times$ $\left.10^{-4}\right) \exp \left(-\frac{t}{660( \pm 40) p s}\right)$. [right] Transient absorbance signal acquired at $450( \pm 2) \mathrm{nm}$, along with an exponential decay fit resulting in the fit equation $\triangle O D=2.9( \pm 0.1) \times$ $10^{-4}+\left(-2.2( \pm 0.1) \times 10^{-4}\right) \exp \left(\frac{t}{1400( \pm 200) p s}\right)$. 


\section{Global Analysis of Transient Absorption Data}

Each TA data set was first cropped to the $425 \mathrm{~nm}-750 \mathrm{~nm}$ range using Surface Xplorer 4.3 .0 software. Background subtraction was also performed using the same software, by subtracting the average of eight to ten spectra acquired at time delays prior to the instrument response. Spectra containing invalid values $(\mathrm{NaN})$ were also removed from the data set by using Surface Xplorer to interpolate between the two neighboring spectra.

Data sets were then analyzed with global rate analysis using Glotaran 1.5.1. All analysis schemes employed a Gaussian instrument response function, a third order polynomial function accounting for dispersion, and a function accounting for coherent artifacts occuring within the instrument response. Analysis schemes also included between two and four kinetic components. Starting values for all parameters were assigned, and the fitting algortithm was then iterated until the residual standard error converged to a constant value within five significant figures.

Fitting TA data sets via global rate analysis returned several outputs. Two associated spectra were returned for each kinetic component, the decay-associated difference spectrum (DADS) and the evolutionassociated difference spectrum (EADS). Both spectra can be analyzed, but neither can be rigorously assigned as a species associated difference spectrum (SADS) that exactly corresponds to the states being probed unless the model is known to be correct a priori. Analysis in this study focuses on the DADS, which correspond more closely to the expected signals of the intermediates in proposed model. Each kinetic component is also associated with an exponential decay function. The fit returns the time constants for each kinetic component, and the DADS for each component is the spectrum of amplitude factors for the corresponding exponential decay.

In the figures below, all uncertainties reported for fitted time constants are standard errors. Time constants reported without a standard error value were fixed to specific values during the fitting procedure. The longest time constant was always fixed to a pseudo-infinite value of $10^{10} \mathrm{ps}$ to account for the long-lived signal that does not decay within the observed domain. The second longest time constant was always fixed to the $S_{1}$ lifetime value measured via time correlated single photon counting (TCSPC). 

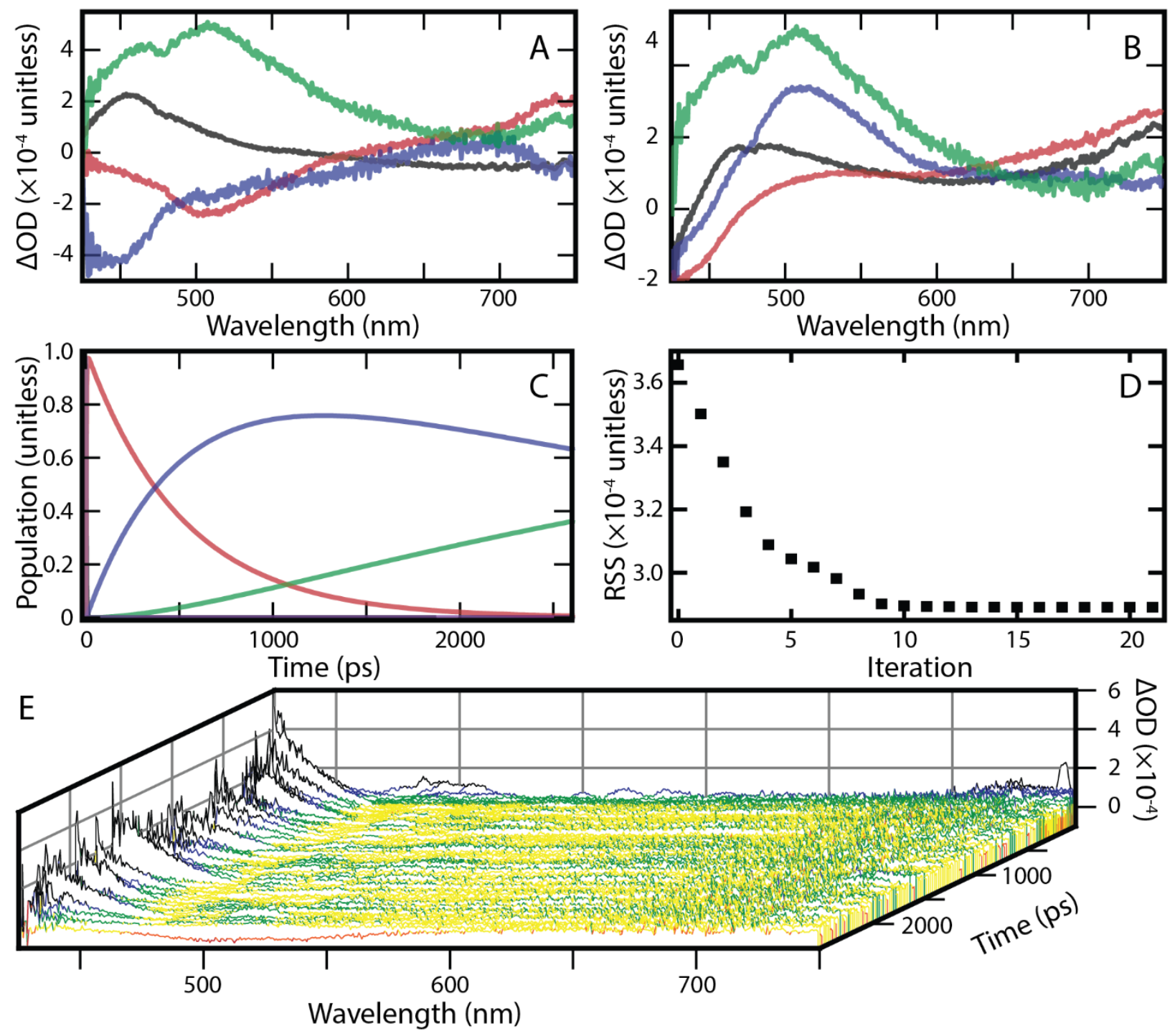

Figure S8: Global Analysis of Transient Absorption Data for Compound 1 in Methanol Solution. The results of global rate analysis of the TA data set are displayed. The results shown were generated with a sequential model employing four kinetic components. Results characterizing the four kinetic components are shown in panels A (DADS), B (EADS), and C (population kinetics). The data for the respective kinetic components is color coded, and each kinetic component corresponds to an exponential decay time constant: $2.67( \pm 0.04)$ ps (black), $520( \pm 10)$ ps (red), 4586 ps (blue), and $10^{10}$ ps (green). Instrument response is also shown in purple in panel $\mathrm{C}$, although both this signal and the 2.67 ps kinetic component overlap strongly with the left and bottom figure axes due to their very fast dynamics. Panel D shows the residual sum of squares for the fit decreasing and then converging on a constant value as the fitting procedure was iterated. Panel E shows the residual of the fit, demonstrating that no significant signals have been excluded from the fit. The color scale used to highlight differences in $\triangle \mathrm{OD}$ is $\left(\right.$ all $\left.\times 10^{-4}\right)$ : -1.0 to -0.6 (red), -0.6 to -0.2 (orange), -0.2 to 0.2 (yellow), 0.2 to 0.6 (green), 0.6 to 1.0 (blue), and $>1.0$ (black). 
TA data for 1 fits best to a model with four kinetic components, shown in Figure S8. Models with three kinetic components return residuals with clear signals remaining, and an $\mathrm{F}$ test at the $95 \%$ confidence level verifies that the model with four components produces a significantly higher quality fit. A model allowing the 4586 ps time constant to vary instead of remaining fixed produced a time constant of $1490( \pm 60)$ ps, along with slight changes to the other time constants, the DADS, and the EADS. An F test showed that the residuals from this model were not significantly different than those from the fixed model.

The DADS for the four components of the model can be assigned in reasonable agreement with the expected properties of 1 . The first DADS, associated with a decay time of $2.67( \pm 0.04) \mathrm{ps}$, has positive amplitude at low wavelengths. Because of its short lifetime, this component is associated with the vibrationally excited $\mathrm{S}_{1}{ }^{*}$ state formed immediately after excitation. The second kinetic component, with a time constant of $520( \pm 10)$ ps, has a DADS characterized by negative amplitude below $\sim 600 \mathrm{~nm}$ and peaking around $505 \mathrm{~nm}$, and positive amplitude at wavelengths above 600 $\mathrm{nm}$. The next kinetic component, with a fixed time constant of $4586 \mathrm{ps}$, has a DADS with a similar shape, but the negative signal peaks around $450 \mathrm{~nm}$ and the positive signal is smaller and peaks near $680 \mathrm{~nm}$. This third component should be closely associated with the vibrationally relaxed $\mathrm{S}_{1}$ state, because the time constant for this component was fixed to the $\mathrm{S}_{1}$ lifetime as measured by TCSPC. Supporting this, the negative signal peaking at $450 \mathrm{~nm}$ is in good agreement with the emission spectrum of $\mathbf{1}$, suggesting $\mathrm{SE}$ from the relaxed $\mathrm{S}_{1}$ state, and the $680 \mathrm{~nm}$ positive peak may correspond to ESA from the $\mathrm{S}_{1} \rightarrow \mathrm{S}_{5}$ transition predicted by DFT calculations at $636 \mathrm{~nm}$. The assignment for the second kinetic component is unclear, but an intermediate relaxation process is occurring on a timescale of roughly 500 ps. The similarity between the DADS of the second and 
third components suggest that an $\mathrm{S}_{1}$-like state is responsible, so this could be further vibrational cooling causing a change in the spectrum. However, the two components demonstrate a blueshifting of the negative stimulated emission signal, where a redshift should be expected. The cause of this discrepancy is currently unclear. The final kinetic component produces a DADS that is positive across the entire domain, with a strong peak at $505 \mathrm{~nm}$ and a shoulder at $460 \mathrm{~nm}$. This peak is assigned as ESA by a triplet state, on the basis of its long lifetime.

Photoexcitation of $\mathbf{1}$ at $350 \mathrm{~nm}$ should produce a vibrationally excited $\mathrm{S}_{1}$ state, with vibrational relaxation expected to be complete within a few ps. The vibrationally relaxed $\mathrm{S}_{1}$ state should then decay via fluorescence, IC, and ISC over a few nanoseconds. Triplet excited states formed via ISC are expected to survive on much longer timescales. The global rate analysis model described above is mostly consistent with this picture, showing evidence of a vibrationally hot $\mathrm{S}_{1}$ state decaying a time constant of $2.67 \mathrm{ps}$ and a long lived excited state that can be comfortably assigned as a triplet. The picture of $\mathrm{S}_{1}$ decay is less clear. Two components with similar DADS and EADS could be assigned as due to $S_{1}$ population. One decays with a fitted time constant of $520( \pm 10) \mathrm{ps}$, while the other has its time constant fixed to the $S_{1}$ lifetime value of $4586 \mathrm{ps}$ measured by TCSPC. One possible assignment is vibrational cooling and solvent rearrangement at different timescales. Based on the time constant, the $\sim 500$ ps relaxation dynamics observed could also be geminate recombination of a charge-separated intermediate, although no such states are known for quinolinones in solution. The long-lived component of the model agrees well with previously observed and unambiguously identified triplet states in quinolinones. 

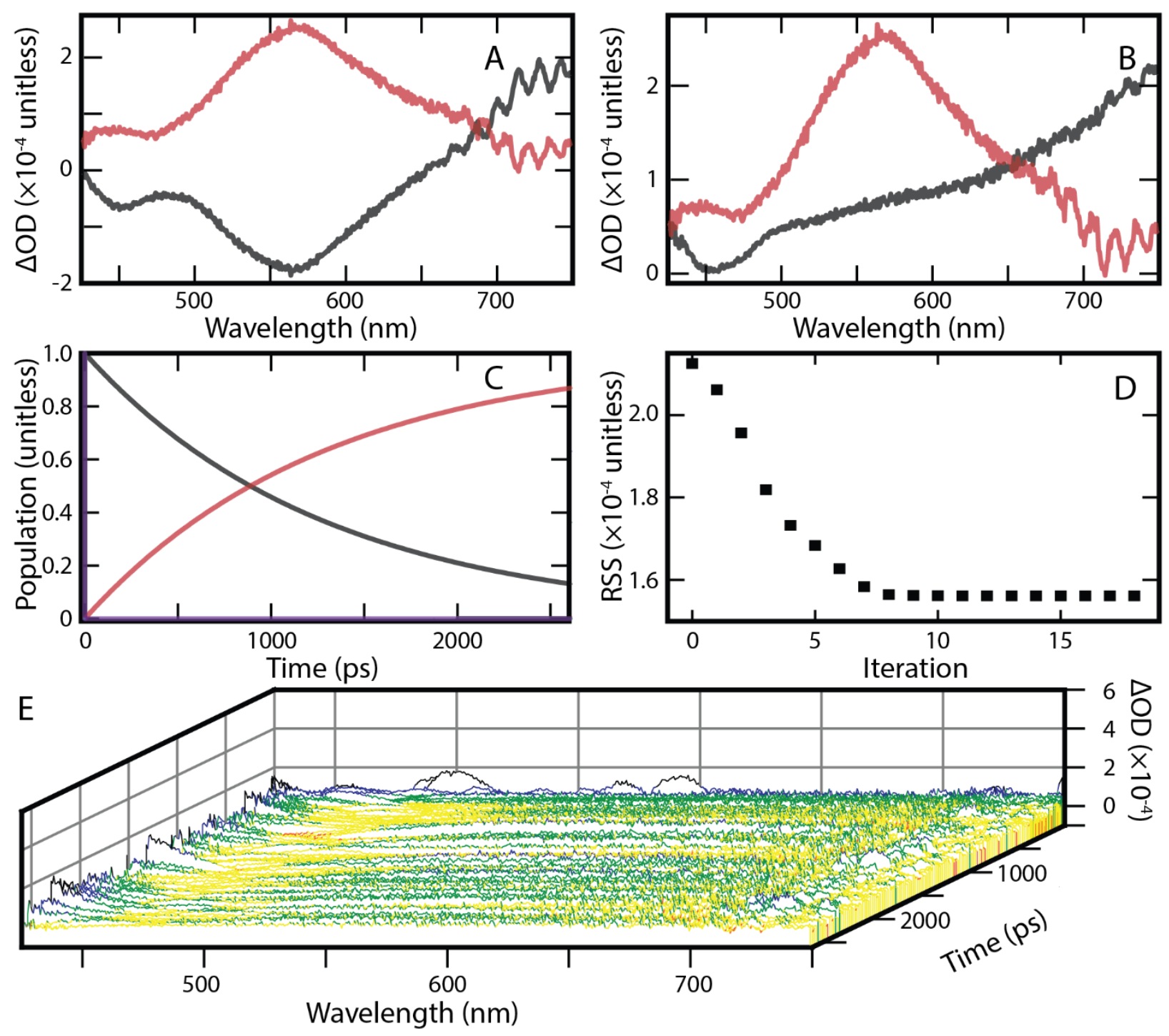

Figure S9: Global Analysis of Transient Absorption Data for Compound 2 in Methanol Solution. The results of global rate analysis of the TA data are displayed. The results shown were generated with a sequential model employing two kinetic components. Results characterizing the two kinetic components are shown in panels A (DADS), B (EADS), and $\mathrm{C}$ (population kinetics). The data for the respective kinetic components is color coded, and each kinetic component corresponds to an exponential decay time constant: $1284 \mathrm{ps}$ (black), and $10^{10} \mathrm{ps}$ (red). Instrument response is also shown in purple in panel $\mathrm{C}$, although the signal overlaps strongly with the left and bottom figure axes due to the very fast dynamics. Panel D shows the residual sum of squares for the fit decreasing and then converging on a constant value as the fitting procedure was iterated. Panel E shows the residual of the fit, demonstrating that no significant signals have been excluded from the fit. The color scale used to highlight differences in $\triangle \mathrm{OD}$ is $\left(\right.$ all $\left.\times 10^{-4}\right):-1.0$ to 0.6 (red), -0.6 to -0.2 (orange), -0.2 to 0.2 (yellow), 0.2 to 0.6 (green), 0.6 to 1.0 (blue), and $>1.0$ (black). 
The data set for $\mathbf{2}$ is best modeled with two kinetic components. The data shown in Figure S9 correspond to global analysis using a two component model, with both time constants fixed. The longer time constant is fixed to $10^{10} \mathrm{ps}$ to account for the long-lived signal in the data set, and the shorter time constant is fixed to the $\mathrm{S}_{1}$ lifetime value of 1284 ps measured via TCSPC. Including a third kinetic component resulted in a model that converged towards an unphysically short time constant, although it does return significantly smaller residuals due to the additional degree of freedom. The residuals shown in Figure S9 show that a small additional feature may be present around $475 \mathrm{~nm}$, but the signal amplitude is small enough that it is not surprising no dynamics could be recovered via modeling. Allowing the 1284 ps time constant to vary produces a time constant of $1220( \pm 10)$ ps. There was no significant difference between the quality of the fits obtained with varying or fixed values for this time constant, so the model with the time constant fixed to the $\mathrm{S}_{1}$ lifetime is preferred.

The DADS associated with the two components of the model are unfortunately strongly mirrored, making them difficult to interpret. This is most likely due to strongly overlapping features in the spectra with competing growth/decay dynamics. No component that could be assigned to vibrational cooling of $\mathrm{S}_{1}{ }^{*}$ was recovered, so no vibrational cooling time is reported for 2. The DADS for the decaying kinetic component was consistent with the expected features of the $\mathrm{S}_{1}$ state. The DADS consists of negative signal peaking at $565 \mathrm{~nm}$ and extending to around 650 $\mathrm{nm}$, and positive amplitude at higher wavelengths. This negative signal overlaps with the static emission spectrum, suggesting an assignment of $\mathrm{SE}$ from the $\mathrm{S}_{1}$ state, but the observed negative signal extends much farther to longer wavelengths. The positive signal is consistent with TDDFT calculations performed at the $\mathrm{S}_{1}$ geometry, which predict transitions at $551 \mathrm{~nm}\left(\mathrm{~S}_{1} \rightarrow \mathrm{S}_{6}\right)$ and 755 $\mathrm{nm}\left(\mathrm{S}_{1} \rightarrow \mathrm{S}_{5}\right)$. The DADS for the long-lived component is positive across the entire domain, and 
peaks at $565 \mathrm{~nm}$. This peak is assigned as ESA by a triplet state, on the basis of its long lifetime (fixed at $10^{10} \mathrm{ps}=0.01 \mathrm{~s}$ ).

The TA data for $\mathbf{2}$ are consistent with the expected picture. The $\mathrm{S}_{1}$ lifetime is well characterized as 1284 ps via TCSPC. The TA data fit well to a model with two decay time constants, one associated with decay of the $S_{1}$ state and using a fixed value of 1284 ps for its time constant, and the other assigned as a long-lived triplet state. Allowing the time constant associated with the $\mathrm{S}_{1}$ feature to vary during fitting did not improve the fit in a statistically significant way. Including a third kinetic component in the model yielded unphysical results, so no component associated with vibrational cooling was recovered from the TA data. This may be due to the blueshifted absorbance spectrum of 2 relative to other compounds, causing less vibrational energy to be imparted during the initial excitation. Alternatively, there may simply be an inherently weaker signal from $\mathrm{S}_{1}{ }^{*}$ in compound $\mathbf{2}$, relative to other molecules studied.

Figure S10 shows the results of global rate analysis of the TA data set for $\mathbf{3}$. The best model of the data set included three kinetic components, two of which had fixed values for their time constants. The longest time constant was fixed to $10^{10} \mathrm{ps}$ to account for the long-lived signal observed, and the middle time constant was fixed to the $S_{1}$ lifetime value of 3018 ps as measured by TCSPC. A model allowing this $\mathrm{S}_{1}$ time constant to vary produced a value of $1690( \pm 50) \mathrm{ps}$, but the quality of the fit was not significantly better than that obtained with a fixed value. Adding a fourth kinetic component to the model recovers an unphysically short time constant of $46( \pm 2)$ fs, and the fit is not significantly improved. 

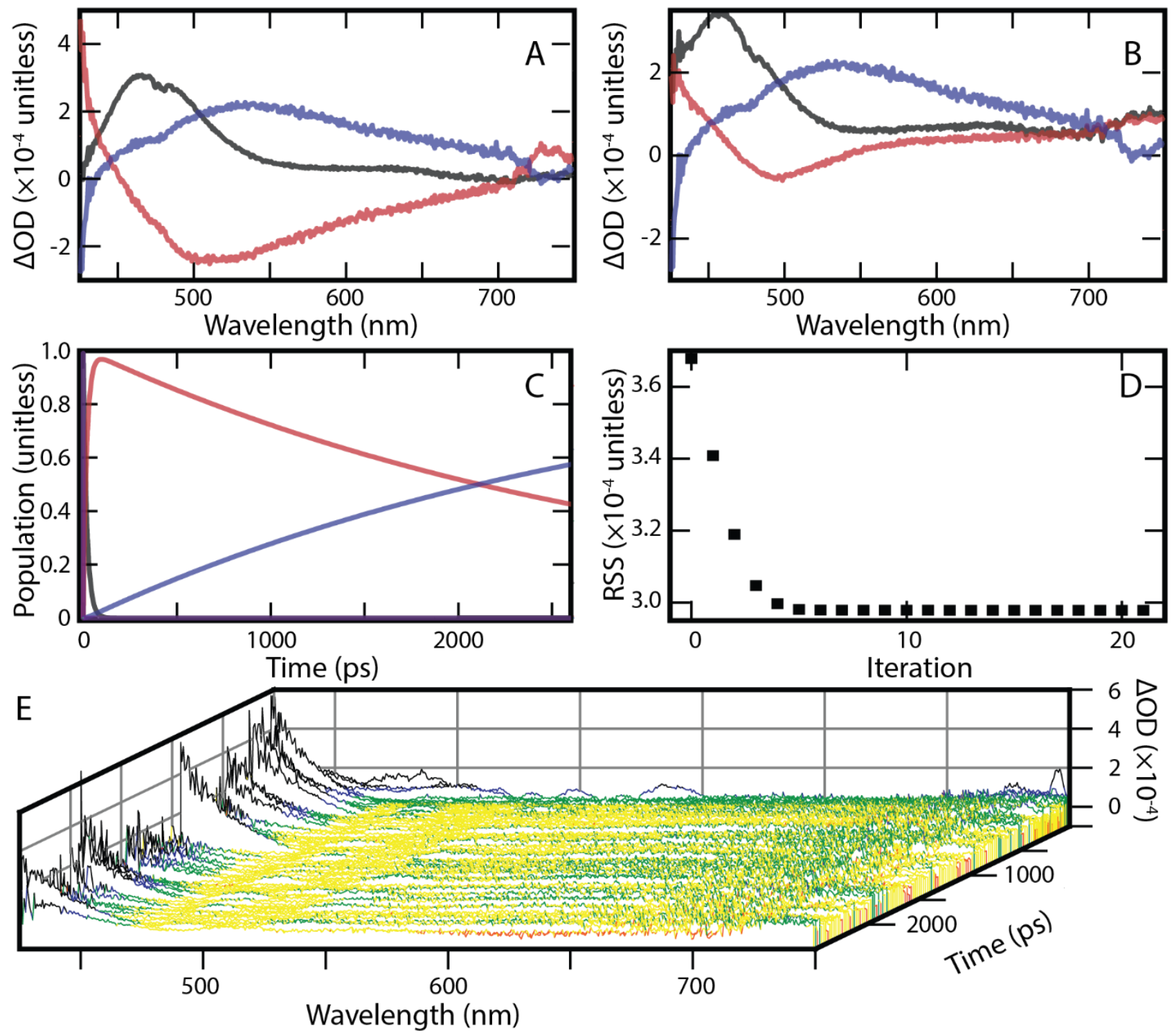

Figure S10: Global Analysis of Transient Absorption Data for Compound 3 in Methanol Solution. The results of global rate analysis of the TA data are displayed. The results shown were generated with a sequential model employing three kinetic components. Results characterizing the three kinetic components are shown in panels A (DADS), B (EADS), and $\mathrm{C}$ (population kinetics). The data for the respective kinetic components is color coded, and each kinetic component corresponds to an exponential decay time constant: 19.3 ( \pm 0.1$)$ ps (black), 3018 ps (red), and $10^{10} \mathrm{ps}$ (blue). Instrument response is also shown in purple in panel $\mathrm{C}$, although the signal overlaps strongly with the left and bottom figure axes due to the very fast dynamics. Panel D shows the residual sum of squares for the fit decreasing and then converging on a constant value as the fitting procedure was iterated. Panel E shows the residual of the fit, demonstrating that no significant signals have been excluded from the fit. The color scale used to highlight differences in $\triangle \mathrm{OD}$ is $\left(\right.$ all $\left.\times 10^{-4}\right):-1.0$ to -0.6 (red), -0.6 to -0.2 (orange), -0.2 to 0.2 (yellow), 0.2 to 0.6 (green), 0.6 to 1.0 (blue), and $>1.0$ (black). 
The DADS associated with $19.3( \pm 0.1)$ ps decay dynamics consists of positive amplitude peaking around $470 \mathrm{~nm}$, as shown in panel A of Figure S10. Based on the dynamics, this feature is assigned as ESA by the initially formed $\mathrm{S}_{1}{ }^{*}$ excited state. The DADS associated with 3018 ps decay is primarily a negative peak near $510 \mathrm{~nm}$. This peak overlaps with the tail of the static emission spectrum, although the negative signal extends much further to long wavelengths, and is assigned as $\mathrm{SE}$ from the relaxed $\mathrm{S}_{1}$ state. Positive amplitude is also observed at the low and high wavelength edges of the spectrum, which is consistent with ESA from $\mathrm{S}_{1}$. TDDFT calculations predict transitions at $444 \mathrm{~nm}\left(\mathrm{~S}_{1} \rightarrow \mathrm{S}_{8}\right)$ and $705 \mathrm{~nm}\left(\mathrm{~S}_{1} \rightarrow \mathrm{S}_{4}\right)$. The DADS representing the long lived component of the model shows positive amplitude peaking around $535 \mathrm{~nm}$. This feature is assigned to ESA by a triplet excited state.

Figure S11 shows results from global rate analysis of TA data for 4 . The best model of the data included three kinetic components, with two having fixed time constants. As in all models discussed in this work, the time constant for the longest kinetic component was fixed to a value of $10^{10}$ ps. The middle kinetic component had a time constant with a fixed value of $1233.7 \mathrm{ps,}$ corresponding to the $\mathrm{S}_{1}$ lifetime as measured by TCSPC. Allowing this time constant to vary produced a value of $1900( \pm 20)$ ps. The unfixed model produces a significantly improved fit, as evaluated by an F test at the $95 \%$ confidence level. However, inspection of the residual shown in panel E of Figure S11 shows that the fixed model does not exclude any substantial dynamic signals. The differences in fit quality are most likely due to a better fit of the noise in the unfixed model, so the fixed model is the preferred result because it includes an accurate value of the $\mathrm{S}_{1}$ lifetime that is certainly driving some of the observed dynamics. Including a fourth kinetic component produces a model that does not converge on a solution. 

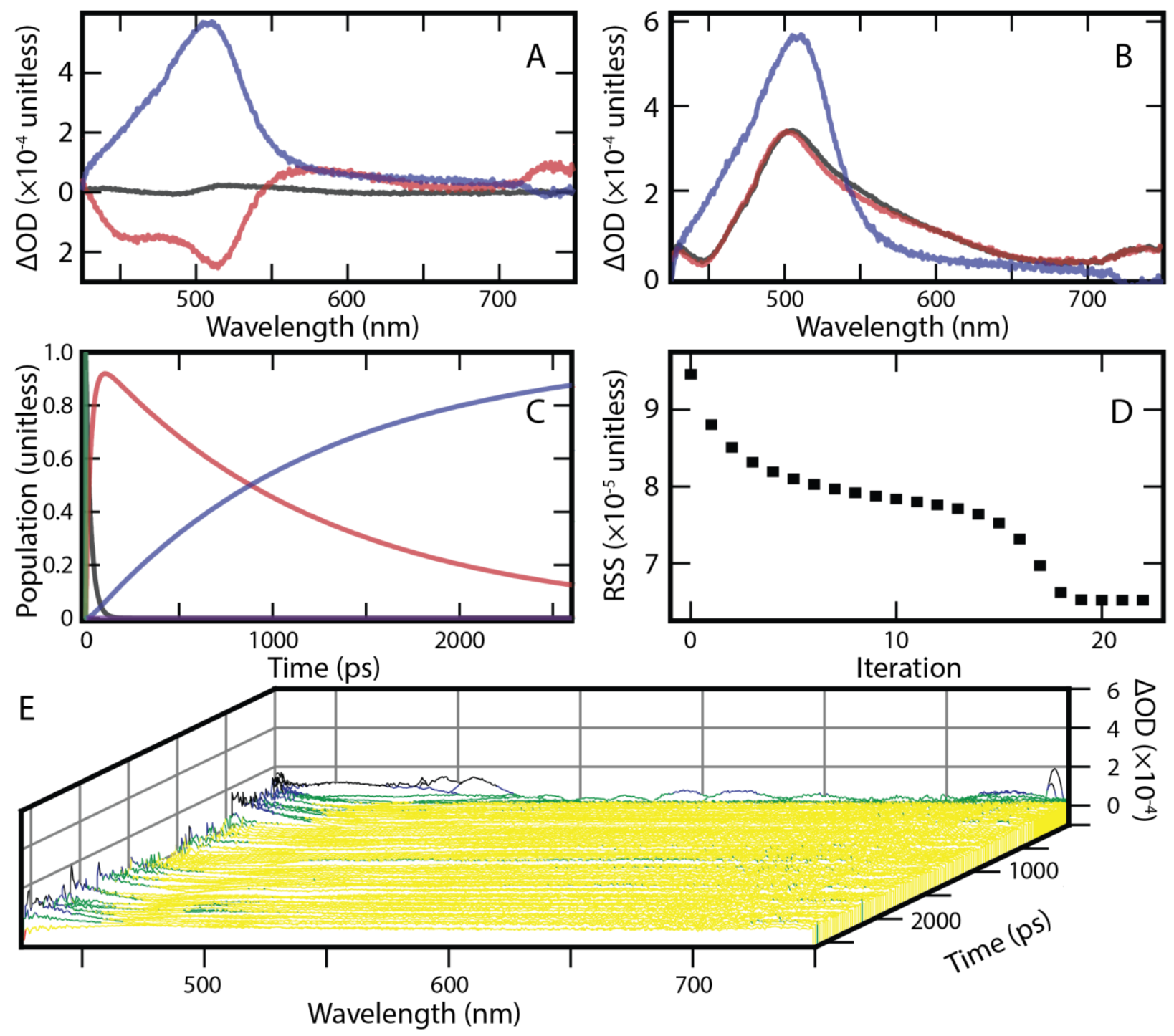

Figure S11: Global Analysis of Transient Absorption Data for Compound 4 in Methanol Solution. The results of global rate analysis of the TA data are displayed. The results shown were generated with a sequential model employing three kinetic components. Results characterizing the three kinetic components are shown in panels A (DADS), B (EADS), and $\mathrm{C}$ (population kinetics). The data for the respective kinetic components is color coded, and each kinetic component corresponds to an exponential decay time constant: 27 ( \pm 1 ) ps (black), 1233.7 ps (red), and $10^{10}$ ps (blue). Instrument response is also shown in purple in panel $\mathrm{C}$, although the signal overlaps strongly with the left and bottom figure axes due to the very fast dynamics. Panel D shows the residual sum of squares for the fit decreasing and then converging on a constant value as the fitting procedure was iterated. Panel E shows the residual of the fit, demonstrating that no significant signals have been excluded from the fit. The color scale used to highlight differences in $\triangle \mathrm{OD}$ is (all $\times 10^{-4}$ ): -1.0 to -0.6 (red), -0.6 to -0.2 (orange), -0.2 to 0.2 (yellow), 0.2 to 0.6 (green), 0.6 to 1.0 (blue), and > 1.0 (black). 
The DADS for the fastest kinetic component, associated with a time constant of $27( \pm 1)$ ps, is consistent with the vibrationally excited $\mathrm{S}_{1}{ }^{*}$ state. This spectrum has very low amplitude, with positive peaks at $437 \mathrm{~nm}$ and $525 \mathrm{~nm}$. These peaks are most likely ESA from the $\mathrm{S}_{1}{ }^{*}$ state. While the amplitude of this signal in the DADS is very small relative to the other spectra derived from the model, including this component in the model does significantly improve the fit.

The DADS for the second kinetic component has a negative signal peaking at $514 \mathrm{~nm}$, with a shoulder at $460 \mathrm{~nm}$, and positive signal above $550 \mathrm{~nm}$. These signals are consistent with the $\mathrm{S}_{1}$ state, validating the choice to fix the time constant for this component to the 1233.7 ps value measured via TCSPC. The negative signal overlaps with the static emission spectrum, although it extends farther to the red side of the spectrum, and is assigned as $\mathrm{SE}$ from $\mathrm{S}_{1}$. The positive signal is assigned as ESA by $\mathrm{S}_{1}$, with TDDFT predicting nearby transitions at $555 \mathrm{~nm}\left(\mathrm{~S}_{1} \rightarrow \mathrm{S}_{8}\right), 598 \mathrm{~nm}$ $\left(\mathrm{S}_{1} \rightarrow \mathrm{S}_{7}\right)$, and $629 \mathrm{~nm}\left(\mathrm{~S}_{1} \rightarrow \mathrm{S}_{6}\right)$

The DADS of the third kinetic component peaks at $507 \mathrm{~nm}$ and is positive across most of the observed domain. This feature is assigned as ESA from a triplet excited state, on the basis of its long time constant, the lack of evidence of photoproduct formation, and similar previous assignments reported in literature.

The observed signals are fully consistent with excitation to the vibrationally excited $\mathrm{S}_{1}{ }^{*}$ state, followed by vibrational relaxation to $S_{1}$ and then subsequent decay of the $S_{1}$ state to the triplet state. Decay of the first component at $27( \pm 1)$ ps provides a good estimate of the timescale of vibrational relaxation, although the DADS spectrum has very low magnitude for this component. The second component of the TA corresponds reasonably well with the expected signals from the $\mathrm{S}_{1}$ state, and fixing the decay time for this component to the $\mathrm{S}_{1}$ lifetime measurement produces a fit as good as any other approach. Assignment of the third component to a triplet state is fairly 
unambiguous, given the similar previously reported signals observed and the lack of any evidence of photoproduct formation.

Global rate analysis for compound $\mathbf{5}$ is shown in Figure S12. The best model for this compound included three kinetic components, with two remaining fixed during the iterative fitting procedure. The longest time constant was fixed to $10^{10} \mathrm{ps}$, as with all other compounds. A second time constant was fixed to the $\mathrm{S}_{1}$ lifetime value of $849.3 \mathrm{ps}$ from the TCSPC measurements. Allowing this time constant to vary during the fit produced a value of $1230( \pm 20)$ ps. This model produced slightly smaller residuals, due to the additional degree of freedom, but inspection of the residuals presented in panel E of Figure S12 shows that the fixed model does not exclude any clear dynamic signals. Including a fourth kinetic component in the model resulted in the fit not converging to a constant residual value. Examining the residuals of the three component model closely shows that there may be a small negative signal excluded from the model at early times around $475 \mathrm{~nm}$. The magnitude of this potential signal is small enough that it can't be captured well by the fitting algorithm.

The DADS for $\mathbf{5}$ don't give much information, because the features corresponding to two of the kinetic components are strongly mirrored. The DADS for the fastest kinetic component has very small magnitude, a positive signal spread broadly across most of the observed domain. The mirrored spectra are the DADS for the 849.3 ps kinetic component and the DADS for the longlived signal. This is likely due to the negative $\mathrm{SE}$ signal from the $\mathrm{S}_{1}$ state in the faster component cancelling out the positive ESA signal from the triplet state. Still, the results are consistent with the picture used to interpret all compounds in this study. 

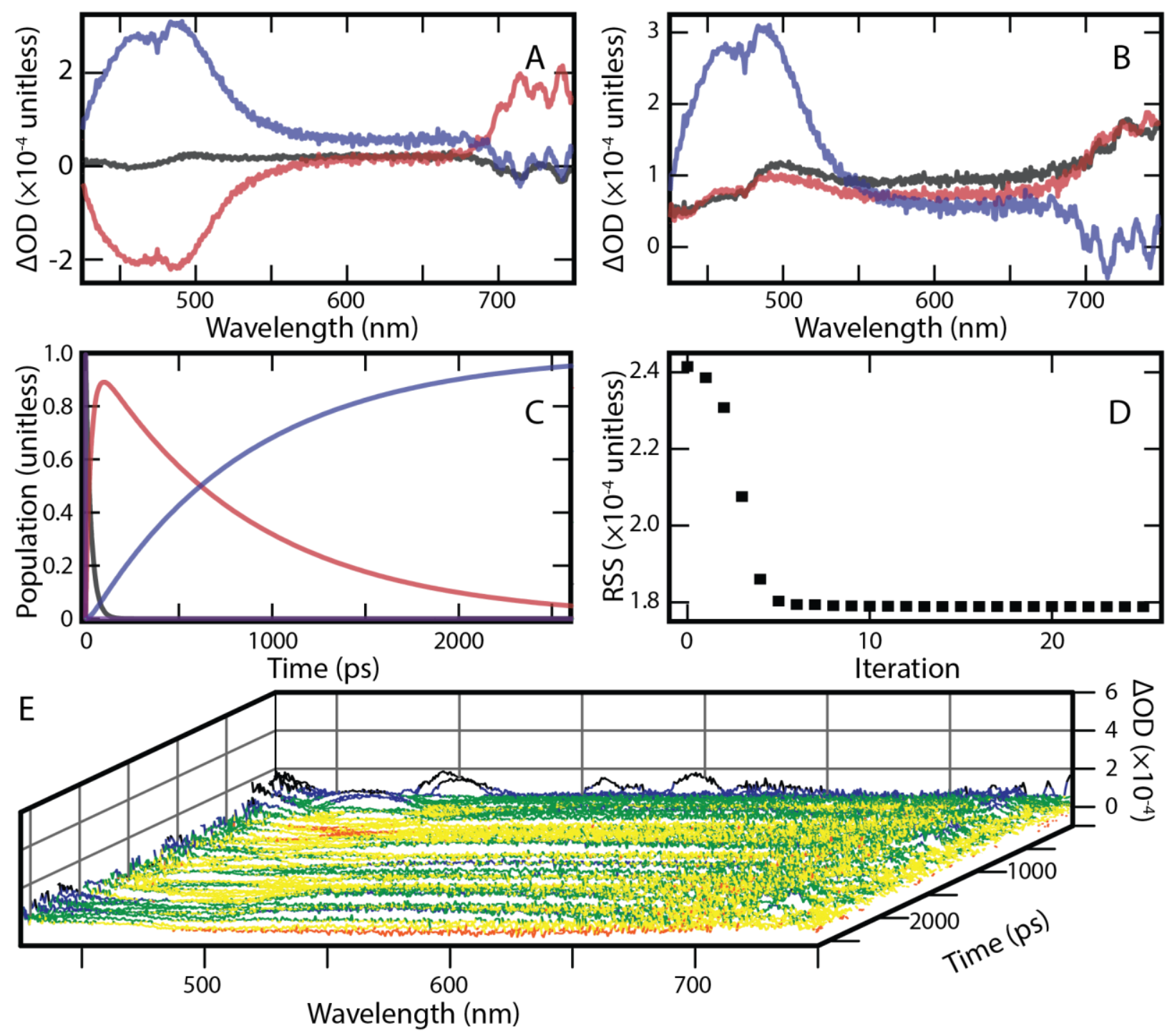

Figure S12: Global Analysis of Transient Absorption Data for Compound 5 in Methanol Solution. The results of global rate analysis of the TA data are displayed. The results shown were generated with a sequential model employing three kinetic components. Results characterizing the three kinetic components are shown in panels A (DADS), B (EADS), and $\mathrm{C}$ (population kinetics). The data for the respective kinetic components is color coded, and each kinetic component corresponds to an exponential decay time constant: 28 ( \pm 1 ) ps (black), 849.3 ps (red), and $10^{10}$ ps (blue). Instrument response is also shown in purple in panel $\mathrm{C}$, although the signal overlaps strongly with the left and bottom figure axes due to the very fast dynamics. Panel D shows the residual sum of squares for the fit decreasing and then converging on a constant value as the fitting procedure was iterated. Panel E shows the residual of the fit, demonstrating that no significant signals have been excluded from the fit. The color scale used to highlight differences in $\triangle \mathrm{OD}$ is $\left(\right.$ all $\times 10^{-4}$ ): -1.0 to -0.6 (red), -0.6 to -0.2 (orange), -0.2 to 0.2 (yellow), 0.2 to 0.6 (green), 0.6 to 1.0 (blue), and $>1.0$ (black). 
An alternative modeling strategy, including simultaneous decay of $S_{1}$ population to both the $S_{0}$ and $\mathrm{T}_{1}$ states, was also investigated. This model included four relaxation processes: $\mathrm{S}_{1}{ }^{*} \rightarrow \mathrm{S}_{1}$, $\mathrm{S}_{1} \rightarrow \mathrm{S}_{0}, \mathrm{~S}_{1} \rightarrow \mathrm{T}_{1}$, and $\mathrm{T}_{1} \rightarrow \mathrm{S}_{0}$. The $\mathrm{T}_{1} \rightarrow \mathrm{S}_{0}$ relaxation process was fixed to a pseudo-infinite time constant, in the same way as described for the sequential model. Unfortunately, this modelling approach did not produce clearer results than the sequential model requiring all $\mathrm{S}_{1}$ population to decay to the $T_{1}$ state. The time constants for the $S_{1} \rightarrow S_{0}$ and $S_{1} \rightarrow T_{1}$ relaxation channels could not be simultaneously varied to fit the data set, and attempts to do so caused the fitting routine to return invalid results. The most likely cause was the overlap of $S_{1}$ and $T_{1}$ signals in the data set. The time constants could be estimated by varying each parameter individually, keeping the others fixed. By iterating a few times, a stable result was produced for all compounds, and values resulting from this process are reported below in Table S13. In a few cases the time constants produced were extremely long, and had very large associated standard errors. Even starting from these values, fitting routines varying $S_{1} \rightarrow S_{0}$ and $S_{1} \rightarrow T_{1}$ relaxation time constants could not produce any valid results for any of the compounds.

\section{Table S13: Time Constants From the Alternative Modeling Strategy}

\begin{tabular}{|l|c|c|c|}
\hline Compound & $\tau_{\mathbf{s}^{*} \rightarrow \mathrm{s} 1}(\mathbf{p s})$ & $\tau_{\mathbf{s} 1 \rightarrow \mathrm{s0}}(\mathbf{p s})$ & $\tau_{\mathbf{s} 1 \rightarrow \mathrm{T} 1}(\mathbf{p s})$ \\
\hline 1 & $2.08( \pm 0.03)$ & $4300( \pm 200)$ & $936( \pm 7)$ \\
\hline 2 & $1.94( \pm 0.04)$ & $13000( \pm 2000)$ & $1180( \pm 10)$ \\
\hline 3 & $18.5( \pm 0.1)$ & $1680( \pm 40)$ & $100000( \pm 400000)$ \\
\hline 4 & $12.8( \pm 0.4)$ & $4330( \pm 90)$ & $3720( \pm 70)$ \\
\hline 5 & $33.2( \pm 0.7)$ & $50000( \pm 30000)$ & $1280( \pm 20)$ \\
\hline
\end{tabular}




\section{Statistical Comparisons of Fit Quality}

Throughout the text in section 5 of this document and the main article, the quality of fit produced by different models of the same TA data set is compared. One method of doing so is to apply an F test, where an F statistic is calculated from the residuals of the fits and compared to a standard value for the corresponding confidence level and number of degrees of freedom. The Glotaran 1.5.1 program returns the root mean square value of the residual for each fit. Squaring this value gives the variance. Because the data sets for each compound were acquired with identical parameters, and the number of fitted parameters is nearly the same and also very small relative to the number of data points recorded, the number of degrees of freedom for each fit is essentially the same. This means the F statistic used to compare any two models can be calculated simply as a ratio of their variances, because the degrees of freedom needed to calculate reduced $\chi^{2}$ values from the variances cancel out. In this study the 95\% confidence level was used for all statistical comparisons. Each TA data set has 309 time delays and 550 wavelength data points for each time delay. The models include two fitted parameters defining the Gaussian instrument response, three fitted parameters defining the dispersion function, and one fitted time constant for each kinetic component. This results in $1.68291 \times 10^{5}$ degrees of freedom for a model with three kinetic components, resulting in an F statistic of 1.0027. Adding or subtracting one kinetic component does not change this value, within the number of digits expressed here.

Ultimately, fit quality is frequently not the determining factor in choosing the best model. In this study there are several cases where the model returning the smallest residuals fails to converge, or converges onto unphysical time constants. There are also several cases where a model that returns smaller residuals is judged to be inferior to one that returns larger residuals, even when the $\mathrm{F}$ test shows that the difference is significant. This is frequently true when comparing "fixed" models, 
with one time constant fixed to the $\mathrm{S}_{1}$ lifetime value measured via TCSPC, to "unfixed" models allowing that time constant to vary. The extra degree of freedom allows the unfixed models to converge to smaller residuals, often producing a statistically significant higher quality of fit. However, proceeding from the knowledge that the $\mathrm{S}_{1}$ state is formed and drives some of the observed dynamics, the fixed model is still preferred. This is especially true in cases where the unfixed model produces a very different time constant, such as in models of $\mathbf{3}$ where the unfixed model yields a time constant of $1690( \pm 45)$ ps. This value is very different from the 3018 ps $\mathrm{S}_{1}$ lifetime measured via TCSPC. When forced to choose between a model with reasonable physics and a slight statistical preference for an alternative model, we select the more physically realistic result.

There are several possible reasons why the unfixed models do not correctly capture the $S_{1}$ lifetimes. First, the time domain observed is shorter than several of the $S_{1}$ lifetimes, and in other cases is only approximately double the lifetime value, so a complete picture of $\mathrm{S}_{1}$ relaxation cannot be captured for most compounds. Second, the SE signal from the $S_{1}$ state strongly overlaps with and cancels the ESA signal from the triplet state. The fitting procedure may not be able to disentangle these competing dynamics. Finally, the $\mathrm{S}_{1}$ state decays through multiple channels, while the model assumes that all $S_{1}$ population decays into triplet population. Fitting the data to a model that decouples the decay of the $\mathrm{S}_{1}$ population from the growth of the triplet population may result in better time constants for the two processes. 


\section{Fitting Results for All Models}

In this section the fitted parameters from all applied models are listed. Only models that converged on constant residual values are included. Not all of these results are discussed in the main text and supporting information, but all results are presented here for completeness. Model type is listed as ' $\mathrm{S}$ ' for sequential or ' $\mathrm{P}$ ' for parallel. The columns labeled $\mathrm{N}_{\mathrm{I}}, \mathrm{N}_{\tau}$, and $\mathrm{N}_{\text {fix }}$ report the number of iterations of the fitting procedure carried out, the total number of kinetic components in the model, and the number of kinetic components with fixed time constants, respectively. The column labelled $\mathrm{RMS}_{\text {res }}$ reports the root mean square of the residuals for the fit, which is a direct output from Glotaran 1.5.1 for each fit. The remaining columns in Table S14 report the time constants resulting from the fit, along with their standard errors. Fixed time constants are reported with standard error values identical to the time constant. 
Table S14: Fitted Time Constants and Residuals

\begin{tabular}{|c|c|c|c|c|c|c|c|c|c|c|c|c|c|}
\hline Molecule & Type & $\mathbf{N}_{\mathbf{l}}$ & $\mathbf{N}_{\boldsymbol{\tau}}$ & $N_{\text {fix }}$ & $\begin{array}{l}R^{R S_{\text {res }}} \\
\left(\times 10^{-5}\right)\end{array}$ & $\tau_{1}(p s)$ & $\begin{array}{l}S_{\mathrm{T} 11} \\
\text { (ps) }\end{array}$ & $\tau_{2}(p s)$ & $\begin{array}{l}\mathrm{SE}_{\mathrm{T2}} \\
\text { (ps) }\end{array}$ & $\begin{array}{c}\tau_{3} \\
\text { (ps) }\end{array}$ & $\begin{array}{l}\mathrm{SE}_{\mathrm{t} 3} \\
\text { (ps) }\end{array}$ & $\begin{array}{c}\tau_{4} \\
\text { (ps) }\end{array}$ & $\begin{array}{l}\mathrm{SE}_{\mathrm{T} 4} \\
\text { (ps) }\end{array}$ \\
\hline 1 & $S$ & 20 & 3 & 1 & 4.1914 & 2.89 & 0.04 & 769 & 5 & $10^{10}$ & $10^{10}$ & $x$ & $x$ \\
\hline 1 & $\mathrm{~S}$ & 23 & 3 & 2 & 4.4529 & 2.00 & 0.03 & 4586 & 4586 & $10^{10}$ & $10^{10}$ & $x$ & $x$ \\
\hline 1 & $\mathrm{~S}$ & 21 & 4 & 2 & 4.1619 & 2.67 & 0.04 & 515 & 10 & 4586 & 4586 & $10^{10}$ & $10^{10}$ \\
\hline 1 & $S$ & 23 & 4 & 1 & 4.1608 & 2.57 & 0.03 & 434 & 10 & 1487 & 62 & $10^{10}$ & $10^{10}$ \\
\hline 1 & $P$ & 16 & 3 & 1 & 4.2884 & 2.87 & 0.04 & 770 & 5 & $10^{10}$ & $10^{10}$ & $x$ & $x$ \\
\hline 1 & $\mathrm{P}$ & 23 & 4 & 1 & 4.2585 & 2.58 & 0.04 & 440 & 11 & 1535 & 67 & $10^{10}$ & $10^{10}$ \\
\hline 1 & $P$ & 23 & 4 & 2 & 4.2595 & 2.68 & 0.04 & 515 & 10 & 4586 & 4586 & $10^{10}$ & $10^{10}$ \\
\hline 2 & $\mathrm{~S}$ & 14 & 2 & 1 & 3.0456 & 1223 & 14 & $10^{10}$ & $10^{10}$ & $x$ & $x$ & $x$ & $x$ \\
\hline 2 & $S$ & 18 & 2 & 2 & 3.0457 & 1284 & 1284 & $10^{10}$ & $10^{10}$ & $x$ & $x$ & $x$ & $x$ \\
\hline 2 & $\mathrm{P}$ & 12 & 2 & 1 & 3.0456 & 1223 & 14 & $10^{10}$ & $10^{10}$ & $x$ & $x$ & $x$ & $x$ \\
\hline 2 & $P$ & 18 & 2 & 2 & 3.0457 & 1284 & 1284 & $10^{10}$ & $10^{10}$ & $x$ & $x$ & $x$ & $x$ \\
\hline 3 & $S$ & 16 & 3 & 1 & 4.2103 & 19.8 & 0.2 & 1691 & 45 & $10^{10}$ & $10^{10}$ & $x$ & $x$ \\
\hline 3 & $S$ & 21 & 3 & 2 & 4.2137 & 19.3 & 0.1 & 3018 & 3018 & $10^{10}$ & $10^{10}$ & $x$ & $x$ \\
\hline 3 & $\mathrm{P}$ & 21 & 3 & 1 & 4.2103 & 19.8 & 0.2 & 1690 & 45 & $10^{10}$ & $10^{10}$ & $x$ & $x$ \\
\hline 3 & $P$ & 21 & 3 & 2 & 4.2137 & 19.3 & 0.1 & 3018 & 3018 & $10^{10}$ & $10^{10}$ & $x$ & $x$ \\
\hline 4 & $\mathrm{~S}$ & 25 & 3 & 1 & 1.9617 & 16.4 & 0.7 & 1904 & 24 & $10^{10}$ & $10^{10}$ & $x$ & $x$ \\
\hline 4 & $S$ & 21 & 3 & 2 & 1.9714 & 27 & 1 & 1233.7 & 1233.7 & $10^{10}$ & $10^{10}$ & $x$ & $x$ \\
\hline 4 & $\mathrm{~S}$ & 21 & 2 & 1 & 1.9728 & 1842 & 19 & $10^{10}$ & $10^{10}$ & $x$ & $x$ & $x$ & $x$ \\
\hline 4 & $\mathrm{~S}$ & 21 & 2 & 2 & 1.9839 & 1233.7 & 1233.7 & $10^{10}$ & $10^{10}$ & $x$ & $x$ & $x$ & $x$ \\
\hline 4 & $P$ & 21 & 3 & 1 & 1.9616 & 16.0 & 0.7 & 1902 & 24 & $10^{10}$ & $10^{10}$ & $x$ & $x$ \\
\hline 4 & $P$ & 21 & 3 & 2 & 1.9713 & 26 & 1 & 1233.7 & 1233.7 & $10^{10}$ & $10^{10}$ & $x$ & $x$ \\
\hline 4 & $P$ & 21 & 2 & 1 & 1.9728 & 1842 & 19 & $10^{10}$ & $10^{10}$ & $x$ & $x$ & $x$ & $x$ \\
\hline 4 & $P$ & 11 & 2 & 2 & 1.9839 & 1233.7 & 1233.7 & $10^{10}$ & $10^{10}$ & $x$ & $x$ & $x$ & $x$ \\
\hline 5 & $S$ & 21 & 2 & 1 & 3.2790 & 1204 & 12 & $10^{10}$ & $10^{10}$ & $x$ & $x$ & $x$ & $x$ \\
\hline 5 & $\mathrm{~S}$ & 21 & 3 & 1 & 3.2556 & 28 & 1 & 1231 & 16 & $10^{10}$ & $10^{10}$ & $x$ & $x$ \\
\hline 5 & $\mathrm{~S}$ & 25 & 3 & 2 & 3.2654 & 28 & 1 & 849.3 & 849.3 & $10^{10}$ & $10^{10}$ & $x$ & $x$ \\
\hline 5 & $P$ & 21 & 2 & 1 & 3.2790 & 1204 & 12 & $10^{10}$ & $10^{10}$ & $x$ & $x$ & $x$ & $x$ \\
\hline 5 & $P$ & 21 & 3 & 1 & 3.2556 & 28 & 1 & 1231 & 16 & $10^{10}$ & $10^{10}$ & $x$ & $x$ \\
\hline 5 & $P$ & 27 & 3 & 2 & 3.2654 & 27 & 1 & 849.3 & 849.3 & $10^{10}$ & $10^{10}$ & $x$ & $x$ \\
\hline
\end{tabular}


In Table S15, additional results for the same set of models are reported. The Type, $\mathrm{N}_{\tau}$ and $\mathrm{N}_{\mathrm{fix}}$ columns have the same definitions as the previous table. The parameters $\mathrm{t}_{0}$ and $\mathrm{W}$ are the time zero and width parameters of the Gaussian instrument response function. The parameter $\lambda_{0}$ is the center wavelength for the dispersion function, and $c_{1}, c_{2}$, and $c_{3}$ are it's coefficients. The $\lambda_{0}$ value is not a fitted parameter, it has a pre-selected constant value.

Table S15: Other Fitted Parameters

\begin{tabular}{|c|c|c|c|c|c|c|c|c|c|c|c|c|c|c|}
\hline Molecule & Type & $\mathbf{N}_{\boldsymbol{\tau}}$ & $N_{\text {fix }}$ & $t_{0}$ (fs) & $\begin{array}{l}\mathrm{SE}_{\mathrm{to}} \\
\text { (fs) }\end{array}$ & $\begin{array}{c}W \\
\text { (fs) }\end{array}$ & SEw (fs) & $\begin{array}{c}\lambda_{0} \\
(\mathrm{~nm})\end{array}$ & c1 & $\mathrm{SE}_{\mathrm{c} 1}$ & c2 & $\mathrm{SE}_{\mathrm{c} 2}$ & c3 & $\mathrm{SE}_{\mathrm{c} 3}$ \\
\hline 1 & $\mathrm{~S}$ & 3 & 1 & 124.201 & 0.7 & 48.7 & 0.3 & 507 & 0.2219 & 0.0006 & 0.036 & 0.001 & -0.0343 & 0.0006 \\
\hline 1 & $S$ & 3 & 2 & 126.696 & 0.8 & 49.5 & 0.3 & 507 & 0.2195 & 0.0006 & 0.036 & 0.001 & -0.0338 & 0.0006 \\
\hline 1 & $S$ & 4 & 2 & 125.119 & 0.7 & 49.0 & 0.3 & 507 & 0.2210 & 0.0005 & 0.035 & 0.001 & -0.0336 & 0.0005 \\
\hline 1 & $S$ & 4 & 1 & 125.279 & 0.7 & 49.0 & 0.3 & 507 & 0.2210 & 0.0005 & 0.035 & 0.001 & -0.0334 & 0.0005 \\
\hline 1 & $P$ & 3 & 1 & 124.3 & 0.7 & 48.9 & 0.3 & 507 & 0.2227 & 0.0006 & 0.035 & 0.001 & -0.0336 & 0.0006 \\
\hline 1 & $P$ & 4 & 1 & 125.089 & 0.7 & 49.1 & 0.3 & 507 & 0.2222 & 0.0005 & 0.034 & 0.001 & -0.0330 & 0.0005 \\
\hline 1 & $P$ & 4 & 2 & 124.688 & 0.7 & 48.9 & 0.3 & 507 & 0.2226 & 0.0005 & 0.034 & 0.001 & -0.0332 & 0.0005 \\
\hline 2 & $S$ & 2 & 1 & 235.376 & 0.3 & 44.7 & 0.2 & 543 & 0.2055 & 0.0006 & 0.0823 & 0.0005 & -0.0720 & 0.0004 \\
\hline 2 & $S$ & 2 & 2 & 235.426 & 0.3 & 44.8 & 0.2 & 543 & 0.2055 & 0.0006 & 0.0824 & 0.0005 & -0.0720 & 0.0004 \\
\hline 2 & $\mathrm{P}$ & 2 & 1 & 235.212 & 0.3 & 44.6 & 0.2 & 543 & 0.2056 & 0.0006 & 0.0822 & 0.0005 & -0.0719 & 0.0004 \\
\hline 2 & $\mathrm{P}$ & 2 & 2 & 235.426 & 0.3 & 44.8 & 0.2 & 543 & 0.2055 & 0.0006 & 0.0824 & 0.0005 & -0.0720 & 0.0004 \\
\hline 3 & $S$ & 3 & 1 & 264.068 & 0.5 & 47.6 & 0.3 & 565 & 0.2073 & 0.0009 & -0.0162 & 0.0005 & -0.0273 & 0.0005 \\
\hline 3 & $S$ & 3 & 2 & 264.146 & 0.5 & 47.7 & 0.3 & 565 & 0.2073 & 0.0009 & -0.0162 & 0.0005 & -0.0273 & 0.0005 \\
\hline 3 & $P$ & 3 & 1 & 264.117 & 0.5 & 47.6 & 0.3 & 565 & 0.2074 & 0.0009 & -0.0164 & 0.0005 & -0.0272 & 0.0005 \\
\hline 3 & $\mathrm{P}$ & 3 & 2 & 264.146 & 0.5 & 47.7 & 0.3 & 565 & 0.2073 & 0.0009 & -0.0162 & 0.0005 & -0.0273 & 0.0005 \\
\hline 4 & $S$ & 3 & 1 & 173.379 & 0.3 & 47.0 & 0.1 & 504 & 0.1995 & 0.0003 & 0.0342 & 0.0006 & -0.0272 & 0.0002 \\
\hline 4 & $S$ & 3 & 2 & 174.132 & 0.3 & 47.0 & 0.1 & 504 & 0.1993 & 0.0003 & 0.0330 & 0.0006 & -0.0266 & 0.0002 \\
\hline 4 & $S$ & 2 & 1 & 174.221 & 0.3 & 47.0 & 0.1 & 504 & 0.1998 & 0.0003 & 0.0325 & 0.0006 & -0.0265 & 0.0002 \\
\hline 4 & $\mathrm{~S}$ & 2 & 2 & 173.77 & 0.3 & 46.9 & 0.1 & 504 & 0.1999 & 0.0003 & 0.0331 & 0.0006 & -0.0268 & 0.0002 \\
\hline 4 & $\mathrm{P}$ & 3 & 1 & 173.373 & 0.3 & 47.0 & 0.1 & 504 & 0.1995 & 0.0003 & 0.0342 & 0.0006 & -0.0272 & 0.0002 \\
\hline 4 & $P$ & 3 & 2 & 173.68 & 0.3 & 47.1 & 0.1 & 504 & 0.1995 & 0.0003 & 0.0337 & 0.0006 & -0.0269 & 0.0002 \\
\hline 4 & $P$ & 2 & 1 & 174.221 & 0.3 & 47.0 & 0.1 & 504 & 0.1998 & 0.0003 & 0.0325 & 0.0006 & -0.0265 & 0.0002 \\
\hline 4 & $P$ & 2 & 2 & 173.779 & 0.3 & 46.9 & 0.1 & 504 & 0.1999 & 0.0003 & 0.0331 & 0.0006 & -0.0268 & 0.0002 \\
\hline 5 & $\mathrm{~S}$ & 2 & 1 & 136.488 & 0.5 & 44.7 & 0.2 & 492 & 0.1016 & 0.0009 & 0.169 & 0.001 & -0.0682 & 0.0005 \\
\hline 5 & $S$ & 3 & 1 & 135.831 & 0.6 & 44.4 & 0.2 & 492 & 0.1015 & 0.0009 & 0.168 & 0.001 & -0.0681 & 0.0005 \\
\hline 5 & $S$ & 3 & 2 & 136.209 & 0.6 & 44.5 & 0.2 & 492 & 0.1009 & 0.0009 & 0.168 & 0.001 & -0.0680 & 0.0005 \\
\hline 5 & $P$ & 2 & 1 & 136.488 & 0.5 & 44.7 & 0.2 & 492 & 0.1016 & 0.0009 & 0.169 & 0.001 & -0.0682 & 0.0005 \\
\hline 5 & $P$ & 3 & 1 & 135.831 & 0.6 & 44.4 & 0.2 & 492 & 0.1015 & 0.0009 & 0.168 & 0.001 & -0.0681 & 0.0005 \\
\hline 5 & $P$ & 3 & 2 & 136.193 & 0.6 & 44.4 & 0.2 & 492 & 0.1008 & 0.0009 & 0.168 & 0.001 & -0.0680 & 0.0005 \\
\hline
\end{tabular}




\section{TCSPC Fitting Results}
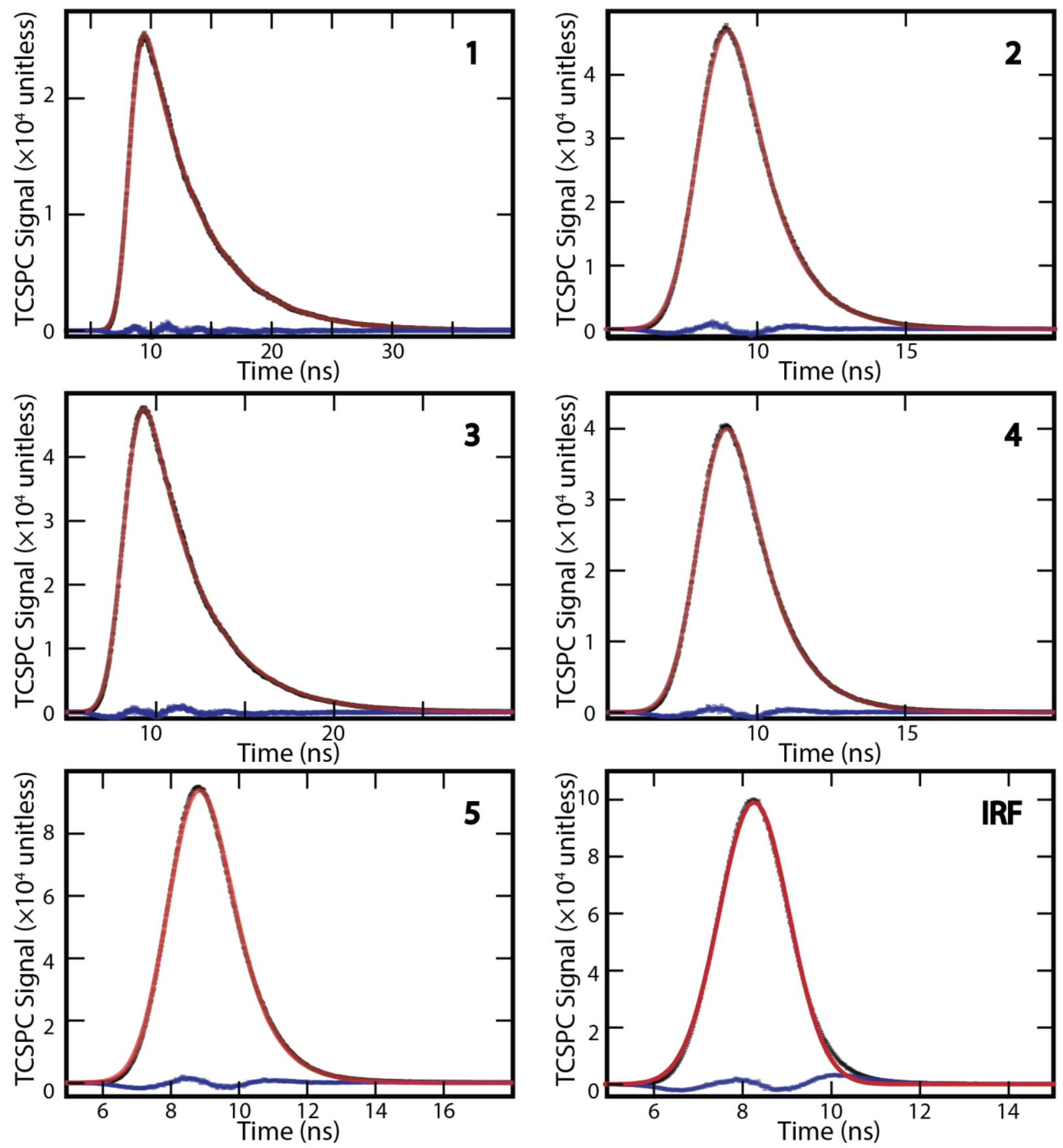

Figure S13: TCSPC Fits and Residuals. Fitting of the time correlated single photon counting data is reported. TCSPC signal is shown in black, with fit lines in red and residuals in blue. 
Figure S13 displays the results of fitting of the TCSPC data. All fits are consistent with monoexponential decay, convoluted with the Gaussian instrument response function. Exponential decay constants from these fits are reported as $\tau_{\mathrm{S} 1}$ in Table 2 . The instrument response fit to a Gaussian function with a standard deviation of $0.7897( \pm 0.0006) \mathrm{ns}$. 